6 Hypothesen zur Prävention von abschlusspolitischen Ergebnisspaltungen mit aufgegebenen Geschäftsbereichen durch interne Corporate-Governance-Mechanismen

Im vorliegenden Kapitel werden aus der Prinzipal-Agenten-Theorie Präventivmaßnahmen für abschlusspolitische Ergebnisspaltungen abgeleitet. Konkret wird untersucht, ob aus theoretischer Sicht die Prävention von abschlusspolitischen Ergebnisspaltungen durch interne Corporate-Governance-Mechanismen in Form von Aufsichtsräten, Vergütungssystemen für Vorstände und Eigentümer- oder Kapitalstrukturen zu vermuten ist. ${ }^{748}$ Am Ende der Abschnitte wird mittels einer Alternativhypothese eine Vermutung für die Prävention von abschlusspolitischen Ergebnisspaltungen postuliert.

\title{
6.1 Überwachungsorgane
}

\subsubsection{Zielsetzung}

Überwachungsorgane wurden in der Tab. 5 als interne Corporate-Governance-Mechanismen klassifiziert, wobei unter den Überwachungsorganen von deutschen Aktiengesellschaften typischerweise deren Aufsichtsräte verstanden werden. Aufsichtsräte sollen insbesondere die Informationsasymmetrien zwischen Aktionären und Vorständen abbauen. ${ }^{749}$ Dahinter steht das Kalkül, dass Aufsichtsräte Vorstände zumeist besser als die individuellen Aktionäre überwachen können. ${ }^{750}$ Bezogen auf die Problemstellung in der vorliegenden Arbeit muss untersucht werden, ob Aufsichtsräte Vorstände von abschlusspolitischen Ergebnisspaltungen abhalten wollen und können. ${ }^{751}$

\subsubsection{Zweistufige Prinzipal-Agenten-Probleme}

Mit Aufsichtsräten, die als zusätzliche Akteure zwischen Aktionären und Vorständen stehen, ergeben sich komplexe Prinzipal-Agenten-Probleme: Die Vorstände werden durch Aufsichtsräte überwacht, die oftmals selbst nur Agenten der Aktionäre sind, da diese häufig nicht persönlich in den Aufsichtsräten vertreten sind. ${ }^{752}$ Daraus ergeben sich zweistufige Prinzipal-Agenten-Probleme, bei denen Aufsichtsräte einerseits als Prinzipale der Vorstän-

748 Allgemeiner Larcker, D. F./Richardson, S. A. (2004), S. 626

749 So Steller, M. (2011), S. 24 f. Allgemeiner Bress, S. (2008), S. 45; Feser, N. (2009), S. 133 f.

750 Vgl. Fama, E. F. (1980), S. 294. Allgemeiner Easterbrook, F. H./Fischel, D. R. (2001), S. 281. Denis, D. K. (2001), S. 208 bemängelt unter Verweis auf Hermalin, B. E./Weisbach, M. S. (2003), S. 10/20, dass bislang nicht formal begründet wurde, warum Überwachungsorgane Prinzipal-Agenten-Probleme zwischen Aktionären und Vorständen effizient eindämmen sollten.

751 Siehe Almaleeh, N. M. S. (2019), S. 32; Böcking, H.-J./Worret, D. (2016), S. 120.

752 Dazu Becht, M./Bolton, P./Röell, A. (2003), S. 13; Feser, N. (2009), S. 15; Heyd, R./Beyer, M. (2001), S. 20 f.; Riedl, A. M. (2008), S. 1.

(C) Der/die Autor(en) 2021

K. Czupalla, Abschlusspolitische Ergebnisspaltungen mit

aufgegebenen Geschäftsbereichen nach IFRS 5, Auditing and

Accounting Studies, https://doi.org/10.1007/978-3-658-34436-8_6 
de und andererseits als Agenten der Aktionäre agieren. ${ }^{753}$ Diese Konstellationen können als Prinzipal-Aufseher-Agenten-Probleme bezeichnet werden, bei denen Aktionären die Rolle der Prinzipale, Aufsichtsräten die Rolle der Aufseher und Vorständen die Rolle der Agenten zukommen. ${ }^{754}$ Vor diesem Hintergrund ist es nicht gesichert, dass Aufsichtsräte Vorstände angemessen überwachen. ${ }^{755}$ Am Willen der Aufsichtsräte, abschlusspolitische Ergebnisspaltungen zu verhindern, kann es vor allem aus zwei Gründen mangeln. Erstens verursacht die Überwachung der Vorstände Arbeitsleid und Freizeiteinbußen, weshalb für opportunistische Aufsichtsräte Anreize bestehen, ihre Rolle als Aufseher zu vernachlässigen. ${ }^{756}$ Zweitens können Vorstände versuchen, Aufsichtsräten Vorteile zu verschaffen, um sich mit den Aufsichtsräten gegen die Aktionäre zu verbünden. Folglich werden Aufsichtsräte nur dann bestrebt sein, Vorstände angemessen zu überwachen, wenn sie sich zum einen anstrengen und zum anderen unabhängig sind. ${ }^{757}$ Als diesbezügliche Anreize kommen vor dem Hintergrund der Prinzipal-Agenten-Theorie einerseits die Haftungs- und Reputationsrisiken der Aufsichtsräte sowie andererseits deren Vergütungsstrukturen in Betracht. ${ }^{758}$ Im Folgenden wird zuerst für Haftungs- und Reputationsrisiken sowie anschließend für Vergütungsstrukturen diskutiert, ob diese für Aufsichtsräte Anreize darstellen, abschlusspolitische Ergebnisspaltungen zu verhindern.

\subsubsection{Haftungs- und Reputationsrisiken}

Die Haftungs- und Reputationsrisiken aus abschlusspolitischen Ergebnisspaltungen ergeben sich für Aufsichtsräte aus deren Pflicht zur Prüfung der Rechnungslegung, ${ }^{759}$ die sich auch auf abschlusspolitische Maßnahmen bezieht. ${ }^{760}$ Obwohl Aufsichtsräte somit grundsätzlich für die Rechnungslegung haften, ${ }^{761}$ kann bezweifelt werden, dass sie aufgrund dieser Haftungsrisiken abschlusspolitische Ergebnisspaltungen verhindern wollen. ${ }^{762} \mathrm{Zum}$ einen kann es im Nachhinein schwer zu beurteilen sein, ob eine verzerrte Rechnungslegung durch Vorstände, die für die Erstellung der Rechnungslegung verantwortlich sind, ${ }^{763}$

753 So Picot, A. et al. (2015), S. 89 f. Vgl. Steller, M. (2011), S. 25; Tebben, T. (2011), S. 52 f.; Velte, P. (2009a), S. 128; Velte, P./Stiglbauer, M. (2011), S. 18.

754 Siehe Khurja, Y. T. v. (2015), S. 39 f.; Tirole, J. (1986), S. 182.

755 Siehe Denis, D. K. (2001), S. 199; Heyd, R./Beyer, M. (2001), S. 20 f.

756 Ähnlich Albersmann, B. T./Hohenfels, D. (2017), S. 150 f. Weiterführend Hermalin, B. E./Weisbach, M. S. (2003), S. $10 / 20$.

757 Hierzu DCGK (2007; 2012), Tz. 5.4.2 Satz 1-3.

758 Gemäß Reimsbach, D. (2011), S. 941 können sich auch ideelle Motive der Aufsichtsräte positiv auf deren Anstrengungen oder Unabhängigkeit auswirken. Da derartige Motive aber mit der Annahme der Prinzipal-Agenten-Theorie, dass Agenten ihre Nutzen opportunistisch zu Lasten der Prinzipale maximieren, im Konflikt stehen können, werden diese hier nicht vertieft.

759 Siehe $\$ 171$ Abs. 1 Satz 1 AktG.

760 So Vetter, E. (2019), §171 AktG, Tz. 5.

761 Siehe $\S 171$ Abs. 1 Satz 1 AktG und $\$ \oint 116$ Satz 1 (i.d.F. vom 19. Juli 2002); 116 Satz 1; 93 Abs. 2 Satz $1 \mathrm{AktG}$.

762 Allgemeiner Tirole, J. (1986), S. $206 \mathrm{f}$.

763 So $\S 91$ AktG. Hierzu Heinhold, M. (1984a), S. 392. 
oder Aufsichtsräte, die für die Prüfung der Rechnungslegung verantwortlich sind, ${ }^{764}$ verursacht wurde. ${ }^{765}$ Zum anderen halten Unternehmen für ihre Aufsichtsräte teilweise Directors \& Officers-Versicherungen (D\&O-Versicherungen) vor, die das Haftungsrisiko der Organmitglieder aus etwaigen Pflichtverletzungen erheblich senken. ${ }^{766}$ Ein stärkerer Anreiz, abschlusspolitische Ergebnisspaltungen verhindern zu wollen, dürfte aus Reputationsverlusten resultieren, die Aufsichtsräten drohen, wenn sie eine exzessive Abschlusspolitik zulassen. ${ }^{767}$ Reputationsverluste können auch ohne juristische Auseinandersetzungen dazu führen, dass Aufsichtsräte abberufen werden oder Schwierigkeiten haben, zukünftig mit verantwortungsvollen Aufgaben betraut zu werden. ${ }^{768}$ Eine allgemeine Definition von Reputation, die sowohl für Individuen als auch für Organisationen verwendet werden kann, ${ }^{769}$ lautet: „Reputation ist eine aus den kollektiven Wahrnehmungen Anderer gebildete wahrgenommene Identität, die die Unklarheit über erwartetes zukünftiges Verhalten reduziert und die komplexe Kombination aus hervorstechenden persönlichen Eigenschaften und Leistungen, demonstriertem Verhalten und beabsichtigten Bildern widerspiegelt, die über einen bestimmten Zeitraum direkt beobachtet und/oder aus sekundären Quellen berichtet werden“770 [Übersetzung d. Verf.]. Der in dieser Definition enthaltene Begriff der Identität kann sich sowohl auf Menschen als auch auf Unternehmen beziehen und kann als Menge von Merkmalen definiert werden, die eine Einheit von einer anderen unterscheidet. $^{771}$

Unabhängig davon, ob nun Haftungs- oder Reputationsrisiken stärkere Anreize für Aufsichtsräte darstellen, sich genug Zeit für kritische Würdigungen der Ergebnisspaltungen zu nehmen und sich dabei nicht von den Vorständen beeinflussen zu lassen, ist das empirische Problem identisch: Sowohl Haftungs- als auch Reputationsrisiken lassen sich als

764 Siehe $\$ 171 \mathrm{Abs}$. 1 Satz $1 \mathrm{AktG}$ hinsichtlich der Prüfung durch Aufsichtsräte sowie $\S 124 \mathrm{Abs} .3$ Satz 1 AktG (i.d.F. vom 2. August 1994; 30. Juli 2009) hinsichtlich des Vorschlags eines Abschlussprüfers und $\S 318$ Abs. 1 Satz 4 HGB hinsichtlich der Auftragserteilung an den Abschlussprüfer. Zu Abschlussprüfungen werden im Kapitel 7 separate Hypothesen entwickelt.

765 Gleichwohl liegt die Beweislast, dass bei der „Geschäftsführung die Sorgfalt eines ordentlichen und gewissenhaften Geschäftsleiters" [ $\S 93 \mathrm{Abs} .1$ Satz $1 \mathrm{AktG}]$ angewendet wurde, gemäß $\S 93 \mathrm{Abs} .2$ Satz 2 AktG sowohl bei Vorständen als auch gemäß $\oint \oint 116$ Satz 1 (i.d.F. vom 19. Juli 2002); 116 Satz 1; 93 Abs. 2 Satz 2 AktG bei Aufsichtsräten.

766 Vgl. Dauner-Lieb, B. (2019b), §93 AktG, Tz. 55-57, die zudem darauf hinweist, dass D\&O-Versicherungen für Organmitglieder auch im Interesse der Aktionäre sein können, da sie wertlose Ansprüche aus der Organhaftung gegen zahlungsunfähige Organmitglieder verhindern.

767 Allgemeiner Holmström, B./Tirole, J. (1989), S. 88. Ähnlich Hermalin, B. E./Weisbach, M. S. (2003), S. 10 .

768 Vgl. Auge-Dickhut, S. (1999), S. 36. Ähnlich Abbott, L. J./Parker, S. (2000), S. 49; Fama, E. F. (1980), S. 294; Fama, E. F./Jensen, M. C. (1983a), S. 315; Koprivica, R. (2009), S. 151. Hierzu auch Ampenberger, $M$. (2010), S. $64 \mathrm{f}$.

769 So Ferris, G. R. et al. (2003), S. 215. Übersichten zu Definitionen der Unternehmensreputation bei Barnett, M. L./Jermier, J. M./Lafferty, B. A. (2006), S. 30-32; Walker, K. (2010), S. 364 f./368.

770 „Reputation is a perceptual identity formed from the collective perceptions of others, which is reflective of the complex combination of salient personal characteristics and accomplishments, demonstrated behavior, and intended images presented over some period of time as observed directly and/or reported from secondary sources, which reduces ambiguity about expected future behavior.“ (Ferris, G. R. et al. (2003), S. 215). Auch Zinko, R. et al. (2007), S. 165; Zinko, R./Rubin, M. (2015), S. 218.

771 Genauso Bromley, D. B. (2001), S. 316. 
Nettogrößen nicht zuverlässig quantifizieren. Zwar könnten die Haftungsrisiken als Bruttobeträge bspw. anhand der Bilanzsummen als Maß für die Unternehmensgröße und anhand der Anzahl der Tochterunternehmen als Maß für die Unternehmenskomplexität grob abgeschätzt werden. Für die konkrete Anreizwirkung auf die Aufsichtsräte dürften jedoch die Nettobeträge von deren individuellen Haftungsrisiken entscheidend sein, die nach einer etwaigen gesamtschuldnerischen Haftung aller Mitglieder ${ }^{772}$ für die individuellen Mitglieder verbleiben und nicht durch deren D\&O-Versicherungen abgedeckt sind. ${ }^{773}$ Allerdings gibt es keine gesetzliche Angabepflicht, ob bzw. in welchem Umfang Unternehmen D\&OVersicherungen für Aufsichtsräte abgeschlossen haben und ob bzw. in welchem Umfang die Organmitglieder private D\&O-Versicherungen abgeschlossen haben. Bei der Quantifizierung von Reputationsrisiken sind diese Probleme sogar noch größer, weil Reputationen abstrakte Konstrukte darstellen, deren Anreizwirkungen auf Aufsichtsräte u. a. davon abhängen, ob sie die Arbeit fortsetzen wollen und an vergleichbaren Aufgaben in anderen Unternehmen interessiert sind. ${ }^{774}$ Es bleibt zu untersuchen, ob Aufsichtsräte durch bestimmte Vergütungsmodelle zu ausreichenden Anstrengungen motiviert und in ihrer Unabhängigkeit bestärkt werden können.

\subsubsection{Vergütungsstrukturen}

Die Ansicht des DCGK, ob variable Vergütungen für Aufsichtsräte sinnvoll und ob für diese ggf. kurz- oder langfristige Bemessungsgrundlagen vorzuziehen sind, hat sich innerhalb des Zeitraums, der in der vorliegenden Arbeit empirisch untersucht wird, grundlegend verändert. Vor dem Jahr 2012 empfahl der DCGK neben festen auch variable Aufsichtsratsvergütungen ${ }^{775}$ und regte an, dass die variablen Vergütungen ,auch auf den langfristigen Unternehmenserfolg bezogene Bestandteile“776 enthalten sollen. Die implizit angeregten erfolgsabhängigen Vergütungen mit kurzfristigen Bemessungsgrundlagen wurde nach massiver Kritik aus der Praxis ${ }^{777}$ ab dem Jahr 2012 aufgegeben und durch die folgende ersetzt: „Wird den Aufsichtsratsmitgliedern eine erfolgsorientierte Vergütung zugesagt, soll sie auf eine nachhaltige Unternehmensentwicklung ausgerichtet sein.“778 Diese Empfehlung lässt eine Distanzierung zu variablen Vergütungen und eine deutliche Distanzierung zu variablen Vergütungen mit kurzfristigen Bemessungsgrundlagen erkennen. Daher wird

772 Siehe $\$ \$ 116$ Satz 1 (i.d.F. vom 19. Juli 2002); 116 Satz 1; 93 Abs. 2 Satz 1 AktG.

773 Gemäß $\$ \S 116$ Satz 1 (i.d.F. vom 19. Juli 2002); 116 Satz 1; 93 Abs. 2 Satz 3 AktG müssen D\&OVersicherungen für Aufsichtsräte, im Gegensatz zu Vorständen, keine Selbstbehalte vorsehen. Verlangen die Satzungen die im DCGK (2007; 2009), Tz. 3.8 Abs. 3 empfohlen Selbstbehalte, dürfen Aufsichtsräte laut Warncke, M. (2010), S. 248 bzw. analog zu Dauner-Lieb, B. (2019b), § 93 AktG, Tz. 64 ihre Haftungsrisiken durch privat abgeschlossene D\&O-Versicherungen weiter senken.

774 Analog Fama, E. F. (1980), S. 301. Hierzu auch Ampenberger, M. (2010), S. 66/69.

775 So DCGK (2008), Tz. 5.4.6 Abs. 2 Satz 1.

776 DCGK (2008), Tz. 5.4.6 Abs. 2 Satz 2.

777 Dazu Bredol, M./Schäfer, H. (2013), S. 652 f.; Werder, A. v./Bartz, J. (2012), S. 876. Exemplarisch MOBOTIX AG (Hrsg.) (2009), S. 33; Roth \& Rau AG (Hrsg.) (2010), S. 18; SNP Schneider-Neureither \& Partner $A G$ (Hrsg.) (2009), S. 38. 
nachfolgend getrennt für variable Vergütungen mit kurzfristigen und sodann mit langfristigen Bemessungsgrundlagen erörtert, ob diese für Aufsichtsräte Anreize bilden, sich genug Zeit für kritische Würdigungen der Ergebnisspaltungen zu nehmen und sich dabei nicht von Vorständen beeinflussen zu lassen.

Variable Vergütungen mit kurzfristigen Bemessungsgrundlagen können für Aufsichtsräte Anreize schaffen, abschlusspolitische Maßnahmen zu tolerieren, da sich diese positiv auf kurzfristige Unternehmenserfolge und somit positiv auf Aufsichtsratsvergütungen auswirken können. ${ }^{779}$ Die Unabhängigkeit der Aufsichtsräte ist vor allem dann gefährdet, wenn kurzfristige Bemessungsgrundlagen der variablen Vergütungen auf Rechnungslegungskennzahlen basieren. Keinesfalls sollten sich kurzfristige Vergütungen der Aufsichtsräte an den Ergebnissen aus den fortgeführten Geschäftsbereichen gemäß IFRS 5.33 Buchst. (b) orientieren, da diese Ergebnisse durch abschlusspolitische Ergebnisspaltungen i. S. d. Abb. 2 erhöht werden können. Ähnliche Fehlanreize können variable Vergütungen, die sich an den Summen aus den Ergebnissen aus den fortgeführten und aufgegebenen Geschäftsbereichen bemessen, in Kombination mit einem Aktienbesitz der Aufsichtsräte setzen. Zwar erhöhen sich in diesen Fällen die unmittelbaren Vergütungen durch abschlusspolitische Ergebnisspaltungen nicht, weil sie die Höhe der aggregierten Jahresergebnisse nicht beeinflussen. Allerdings können die Kurse der Aktien der Aufsichtsräte steigen, wenn die Kapitalmärkte nicht erkennen, dass die Ergebnisse aus den fortgeführten Geschäftsbereichen durch abschlusspolitische Ergebnisspaltungen erhöht wurden. Ebenso würden in diesen Fällen Aufsichtsräte profitieren, bei denen die Börsenkurse als Parameter für variable Vergütungen dienen. ${ }^{780}$ Insofern tolerieren Aufsichtsräte abschlusspolitische Ergebnisspaltungen möglicherweise auch dann, wenn die kurzfristigen Bemessungsgrundlagen der variablen Vergütungen nicht auf Rechnungslegungskennzahlen basieren.

Variable Aufsichtsratsvergütungen mit langfristigen Bemessungsgrundlagen ${ }^{781}$ können grundsätzlich dazu beitragen, dass Aufsichtsräte die strategischen Entscheidungen der Vorstände besonders kritisch hinterfragen und abschlusspolitische Maßnahmen verhindern. Langfristig werden abschlusspolitische Maßnahmen mithin unattraktiver, da sie sich regelmäßig umkehren. ${ }^{782}$ Auch abschlusspolitische Ergebnisspaltungen werden langfristig unattraktiver, da regelmäßig anfallende Aufwendungen aus den fortgeführten Geschäftsbereichen, die im aktuellen Geschäftsjahr abschlusspolitisch den aufgegebenen Geschäftsbereichen zugeordnet werden, spätestens im ersten Geschäftsjahr nach den vollständigen Abwicklungen der aufgegebenen Geschäftsbereiche wieder in die Ergebnisse aus den fort-

779 Allgemeiner Pfleger, G. (1991), S. 25.

780 Gemäß Henssler, M. (2019), § 113 AktG, Tz. 12 wären derartige Vergütungsstrukturen aber nur zulässig, wenn der Aktienkurs lediglich einen von mehreren Parametern der variablen Vergütungen bilden würde.

781 Beispielsweise entsprechend DCGK (2012), Tz. 5.4.6 Abs. 2 Satz 2.

782 Dazu Ruhnke, K./Simons, D. (2018), S. 364. 
geführten Geschäftsbereichen zurückkehren. ${ }^{783}$ Somit dürften variable Vergütungen mit langfristigen Bemessungsgrundlagen für Aufsichtsräte grundsätzlich einen Anreiz bilden, abschlusspolitische Ergebnisspaltungen zu verhindern.

\subsubsection{Kollusionen zwischen Aufsichtsräten und Vorständen}

Unabhängig davon, ob variable Aufsichtsratsvergütungen kurz- oder langfristige Bemessungsgrundlagen haben, können sie im Extremfall ein kollusives Zusammenwirken zwischen Vorständen und Aufsichtsräten ${ }^{784}$ begünstigen, da variable Aufsichtsratsvergütungen u. a. von den strategischen Entscheidungen der Vorstände determiniert werden. ${ }^{785} \mathrm{Ganz}$ allgemein können Kollusionen als „Zusammenwirken Mehrerer zum Nachteil Dritter“786 definiert werden. Bezogen auf die im Unterabschnitt 6.1.2 beschriebenen zweistufigen Prinzipal-Agenten-Probleme zwischen Aktionären, Aufsichtsräten und Vorständen können Kollusionen darin bestehen, dass sich Vorstände und Aufsichtsräte zu gegenseitigen Vorteilen verhelfen, die für Aktionäre jedoch nachteilig sind. ${ }^{787}$ Beispielsweise könnten Aufsichtsräte abschlusspolitische Ergebnisspaltungen zugunsten der Vorstände tolerieren, wenn die Vorstände im Gegenzug Unternehmensstrategien umsetzen, ${ }^{788}$ die den Vergütungsinteressen der Aufsichtsräte zugute kommen. ${ }^{789}$ Die gefährdete Unabhängigkeit der Aufsichtsräte aufgrund von variablen Vergütungen ließe sich durch aufwandsbezogene Vergütungen vermeiden, bei denen Aufsichtsräte pro Zeiteinheit, die sie für die Überwachung der Vorstände aufbringen, vergütet werden. ${ }^{790}$ Da Aktionäre aber nicht beobachten können, wie viel Zeit Aufsichtsräte außerhalb von Sitzungen für die Vorstandsüberwachung aufbringen, scheitern anreizoptimale variable Aufsichtsvergütungen an HiddenActions- und Hidden-Informations-Problemen. ${ }^{791}$ Ebenso problematisch sind Vergütungen, die Kollusionen zwischen Vorständen und Aufsichtsräten verhindern sollen, indem deren variable Vergütungen von unterschiedlichen Parametern abhängig gemacht werden. Dann besteht die Gefahr, dass Aufsichtsräte strategische Vorhaben der Vorstände blockie-

783 Siehe den Unterabschnitt 4.3.3.

784 Dazu allgemein Auge-Dickhut, S. (1999), S. 141-155; Tirole, J. (1992), S. 152.

785 Siehe Khurja, Y. T. v. (2015), S. 86. Analog Böcking, H.-J. (2008), S. 370.

786 Grützner, T./Jakob, A. (2015), Stichwort „Kollusion“.

787 Vgl. Holmström, B./Tirole, J. (1989), S. 88.

788 Vgl. dazu $D C G K$ (2008), Tz. 3.2/4.1.2.

789 Ähnlich MOBOTIX AG (Hrsg.) (2009), S. 33; Roth \& Rau AG (Hrsg.) (2010), S. 18; SNP SchneiderNeureither \& Partner AG (Hrsg.) (2009), S. 38. Beispielsweise schreibt die SNP Schneider-Neureither \& Partner AG (Hrsg.) (2009), S. 38 in ihrem Geschäftsbericht für 2008: „Die Gesellschaft gewährt ihren Aufsichtsräten eine angemessene Festvergütung und hat sich gegen eine Aufspaltung dieser Festvergütung in feste und variable Komponenten entschieden, da letztere - wenn auch oftmals theoretisch - das Risiko bergen, dass Entscheidungen des Aufsichtsrats nicht ausschließlich zum Wohl der Gesellschaft, sondern auch mit der Zielrichtung, auf eine Erhöhung von variablen Vergütungskomponenten hinzuwirken, getroffen werden könnten."

790 Vgl. Böcking, H.-J. (2008), S. 370/374. Ungefähr auch Reimsbach, D. (2011), S. 944.

791 Allgemeiner Böcking, H.-J. (2008), S. 370 f. Siehe zu Hidden-Actions- und Hidden-InformationsProblemen die Tab. 4. 
ren, die zwar i.S.d. Aktionäre wären, aber den Vergütungsinteressen der Aufsichtsräte entgegenstehen. ${ }^{792}$

\subsubsection{Zwischenfazit}

In den vorherigen Unterabschnitten wurde untersucht, ob Aufsichtsräte Vorstände von abschlusspolitischen Ergebnisspaltungen abhalten wollen. Als Zwischenfazit ist festzuhalten, dass variabel vergütete Aufsichtsräte abschlusspolitische Maßnahmen der Vorstände möglicherweise tolerieren oder sogar davon profitieren. Eine naheliegende Lösung stellen ausschließlich fixe Aufsichtsratsvergütungen dar. ${ }^{793}$ Allerdings resultiert aus ausschließlich fixen Vergütungen für opportunistische Aufsichtsräte der Anreiz, ihre Anstrengungen zu minimieren. ${ }^{794}$ Deshalb müssen Aufsichtsräte zusätzlich Haftungs- oder Reputationsrisiken ausgesetzt sein, damit sie abschlusspolitische Ergebnisspaltungen unterbinden wollen. Da sich diese Haftungs- oder Reputationsrisiken empirisch nicht zuverlässig quantifizieren lassen, wird in der vorliegenden Arbeit vereinfachend angenommen, dass diese zumindest für die Aufsichtsräte von kapitalmarktorientierten Unternehmen ausreichend hoch sind. ${ }^{795}$ In den folgenden Unterabschnitten wird untersucht, ob Aufsichtsräte Vorstände von abschlusspolitischen Ergebnisspaltungen abhalten können.

\subsubsection{Qualifikationen}

Damit Aufsichtsräte abschlusspolitische Ergebnisspaltungen verhindern können, müssen sie abschlusspolitische Ergebnisspaltungen erkennen können. ${ }^{796}$ Neben den im relevanten Zeitraum von 2008 bis 2013 eingeführten gesetzlichen Vorschriften zur Qualifikation von Aufsichtsräten, ${ }^{797}$ können Aktionäre weitgehend selbst bestimmen, über welche Fachkenntnisse die Aufsichtsräte ihrer Unternehmen verfügen, da sie diese größtenteils selbst wählen. ${ }^{798}$ Zwar sind den Aktionären vor der Bestellung von neuen Aufsichtsräten

792 Zum Vorhergehenden Mertens, H.-J./Cahn, A. (2012), § 113 AktG, Tz. 18.

793 Im Ergebnis auch Perlet im Interview von Wadewitz, S. (2016), S. 9.

794 Allgemeiner Böcking, H.-J. (2008), S. 370 f.

795 Vgl. Auge-Dickhut, S. (1999), S. 36; Faber, J. (2009), S. 226; Favoccia, D./Thorborg, H. (2017), S. 8; Koch, J. (2020), § 116 AktG, Tz. 1/13. Tödtmann, C. et al. (2016), S. 58-60 zeigen mit Praxisbeispielen auf, dass Haftungsrisiken für Aufsichtsräte von kapitalmarktorientierten Unternehmen bedeutsamer werden.

796 Fälle, in denen einzelne Organmitglieder abschlusspolitische Ergebnisspaltungen nicht verhindern können, da sie sich gegen Mitglieder mit einer anderen Ansicht oder mit beeinträchtigter Unabhängigkeit nicht durchsetzen können, werden in der vorliegenden Arbeit nicht beleuchtet. Hierzu AugeDickhut, S. (1999), S. 36; Schewe, G. (2018), S. 37; Warncke, M. (2010), S. 237.

797 Der relevante Untersuchungszeitraum für Hypothesen, die auf gesetzlichen Regelungen beruhen, wird im Abschnitt 8.1 abgegrenzt. Beispielsweise wurde für kapitalmarktorientierte Gesellschaften, die in der vorliegenden Arbeit empirisch untersucht werden, durch $\S 100$ Abs. 5 AktG (i.d.F. vom 25. Mai 2009) eingeführt, dass „mindestens ein unabhängiges Mitglied des Aufsichtsrats über Sachverstand auf den Gebieten Rechnungslegung oder Abschlussprüfung verfügen“ muss. Zuvor enthielt das Aktiengesetz keine vergleichbare Regelung.

798 So $\$ 101$ Abs. 1 Satz 1 AktG. Hierzu Koprivica, R. (2009), S. 41. Ausnahmen bilden bspw. die ebenfalls in $\S 101 \mathrm{Abs}$. 1 Satz $1 \mathrm{Akt} G$ genannten Gesetze zu den Mitbestimmungsrechten von Arbeitnehmern. 
deren tatsächliche Fachkenntnisse typischerweise unbekannt. ${ }^{799}$ Allerdings können diese Hidden-Characteristics-Probleme mit den in der Tab. 4 genannten Lösungsansätzen eingedämmt werden. Insbesondere durch Signalings dürften sich Qualifikationen relativ leicht abschätzen lassen, da für potenzielle Aufsichtsräte, die bspw. dem Berufsstand der Steuerberater oder Wirtschaftsprüfer angehören, angenommen werden kann, dass sie abschlusspolitische Ergebnisspaltungen grundsätzlich erkennen können. Hinsichtlich der empirischen Untersuchung ergibt sich jedoch das Problem, dass weder eine gesetzliche Pflicht noch eine Empfehlung des DCGK existiert, die Qualifikationen der Aufsichtsräte detailliert zu veröffentlichen. ${ }^{800}$ Zwar müssen Kapitalgesellschaften in Deutschland die ausgeübten Berufe ihrer Aufsichtsräte und börsennotierte Gesellschaften zusätzlich die Mitgliedschaften ihrer Aufsichtsräte in anderen Aufsichtsräten und Kontrollgremien angeben. ${ }^{801}$ Die genauen Qualifikationen der Aufsichtsräte lassen sich daraus jedoch nicht zuverlässig ableiten, da bspw. deren Ausbildungen, Berufserfahrungen oder Verantwortung nicht angegeben werden müssen und insbesondere die beiden letztgenannten Faktoren nur schwer zu quantifizieren wären.

\subsubsection{Prüfungsausschüsse}

Möglicherweise können Aufsichtsräte abschlusspolitische Ergebnisspaltungen besser erkennen, wenn sie Ausschüsse bilden, die auf die Überwachung der Rechnungslegung spezialisiert sind. ${ }^{802}$ Ausschüsse dienen grundsätzlich dazu, Verhandlungen und Beschlüsse des Aufsichtsrats vorzubereiten oder die Ausführung seiner Beschlüsse zu überwachen. ${ }^{803}$ Um abschlusspolitische Ergebnisspaltung zu verhindern, erscheinen Prüfungsausschüsse besonders geeignet, ${ }^{804}$ da sich diese „,mit der Überwachung des Rechnungslegungsprozesses, der Wirksamkeit des internen Kontrollsystems, des Risikomanagementsystems und des internen Revisionssystems sowie der Abschlussprüfung, hier insbesondere der Auswahl und der Unabhängigkeit des Abschlussprüfers und der vom Abschlussprüfer zusätzlich erbrachten Leistungen “ 805 befassen. Gesetzliche Pflichten zur Bildung vom Prüfungsausschüssen bestehen für Aktiengesellschaften - auch wenn diese kapitalmarktorientiert sind - nicht. ${ }^{806}$

799 Vgl. Tirole, J. (2006), S. 336.

800 Dazu Böcking, H.-J. (2008), S. 366 f./374, der daher vorschlägt, die Qualifikationen auf den Internetseiten der Unternehmen anzugeben.

801 So $\S 285 \mathrm{Nr}$. 10 Satz 1 HGB (i.d.F. vom 3. August 2005; 31. Juli 2009).

802 Konkret Böcking, H.-J./Worret, D. (2016), S. 120. Allgemeiner Böcking, H.-J. (2008), S. 359; Deckert, M. (1996), S. 989; Steller, M. (2011), S. 103.

803 So $\$ 107$ Abs. 3 Satz 1 AktG.

804 Siehe Böcking, H.-J./Worret, D. (2016), S. 120. Sehr zurückhaltend Forster, K.-H. (1995), S. 6.

$805 \S 107 \mathrm{Abs}$. 3 Satz $2 \mathrm{AktG}$. Allerdings weist $\$ 107 \mathrm{Abs}$. 3 Satz 2 AktG (i.d. F. vom 25. Mai 2009) erst ab dem Inkrafttreten am 29. Mai 2009 auf diesen Aspekt hin.

806 So Habersack, M. (2019), § 107 AktG, Tz. 111; Hambloch-Gesinn, S./Gesinn, F.-J. (2017), § 107 AktG, Tz. 105; Spindler, G. (2019a), § 107 AktG, Tz. 140; Tomasic, L. (2013), § 107 AktG, Tz. 42. 
Prüfungsausschüsse sollen insbesondere etwaige Überwachungsdefizite hinsichtlich der Rechnungslegung verhindern. ${ }^{807}$ Aus der Perspektive der Prinzipal-Agenten-Theorie lösen Prüfungsausschüsse die Prinzipal-Agenten-Probleme zwischen Aufsichtsräten als Prinzipalen und Vorständen als deren Agenten jedoch nicht, sondern ersetzen sie durch weitere Prinzipal-Agenten-Probleme, die sich möglicherweise besser eindämmen lassen. ${ }^{808}$ Wie bereits bei Aufsichtsräten, welche die Prinzipal-Agenten-Probleme zwischen Aktionären und Vorständen eindämmen sollen, gleichzeitig aber zusätzliche Prinzipal-AgentenProbleme zwischen den Aktionären und Aufsichtsräten verursachen, werden mit Prüfungsausschüssen weitere Prinzipal-Agenten-Probleme mit den Aufsichtsräten als Prinzipalen und den Prüfungsausschüssen als deren Agenten kreiert. ${ }^{809}$ Die Reihungen der PrinzipalAgenten-Probleme zwischen Aktionären, Aufsichtsräten, Prüfungsausschüssen und Vorständen bringen folglich dreifache Prinzipal-Agenten-Probleme hervor. ${ }^{810}$ Dabei sollen alle Instanzen, die zwischen die Aktionäre und Vorstände geschaltet werden, die PrinzipalAgenten-Probleme zwischen den beiden vorherigen Instanzen eindämmen. Folglich können Aufsichtsräte aufgrund von Prüfungsausschüssen abschlusspolitische Ergebnisspaltungen nur dann besser erkennen, wenn diese Gremien die Rechnungslegung effizienter und effektiver ${ }^{811}$ überwachen können als die gesamten Aufsichtsräte. ${ }^{812}$ Hierfür sprechen zwei Argumente. Erstens sind die Anforderungen an Aufsichtsräte u. a. aufgrund der zunehmenden Komplexität der internationalen Rechnungslegungsvorschriften erheblich gestiegen. ${ }^{813}$ Aufgrund ihrer geringeren Mitgliederzahlen können Prüfungsausschüsse die Rechnungslegung typischerweise flexibler, intensiver und zeitnäher überwachen als die Plenen der Aufsichtsräte. ${ }^{814}$ Zweitens können Prüfungsausschüsse die Zusammenarbeit zwischen einerseits den Aufsichtsräten sowie andererseits den Abschlussprüfern und den internen Revisionen professionalisieren, ${ }^{815}$ wenn der Empfehlung des DCGK gefolgt wird, dass die Vorsitzenden der Prüfungsausschüsse ,über besondere Kenntnisse und Erfahrun-

807 Vgl. AKEIÜ (Hrsg.) (2000), S. 2283; Böcking, H.-J. (2008), S. 360; Forster, K.-H. (1995), S. 5; Lück, W. (1999), S. 442 f.; Quick, R./Höller, F./Koprivica, R. (2008), S. 25; Steller, M. (2011), S. 26 f.

808 Sinngemäß Deckert, M. (1996), S. 987.

809 Potenzielle Nachteile werden im Detail bei Fischbach, D. (2003), S. 103 f. diskutiert.

810 Etwa Böcking, H.-J./Dutzi, A./Müßig, A. (2004), S. 424 f.; Deckert, M. (1996), S. 990.

811 Beispielsweise stellen Fischbach, D. (2003), S. 102; Kram, L. K. (2012), S. 38; Lück, W. (1999), S. 443; Pfitzer, N./Höreth, U. (2005), S. 175; Spindler, G. (2015a), § 107 AktG, Tz. 140 die Effizienz sowie bspw. AKEIÜ (Hrsg.) (2000), S. 2282; Böcking, H.-J./Dutzi, A./Müßig, A. (2004), S. 426; Deckert, M. (1996), S. 987/989; Faber, J. (2009), S. 227; Marten, K.-U./Quick, R./Ruhnke, K. (2020), S. 73; Quick, R./Höller, F./Koprivica, R. (2008), S. 25; Steller, M. (2011), S. 111 sowohl die Effizienz als auch die Effektivität in den Vordergrund. Koprivica, R. (2009), S. 34-51 definiert und diskutiert Effizienz als „die Relation von Zielbeitrag und der dazu benötigten Ressourcen“ und Effektivität als „die Eignung einer Maßnahme, das verfolgte Ziel zu erreichen“.

812 Vgl. Albersmann, B. T./Hohenfels, D. (2017), S. 150-153; Spindler, G. (2019a), § 107 AktG, Tz. 140. Weiterführend Forster, K.-H. (1995), S. 6 f.

813 Vgl. Albersmann, B. T./Hohenfels, D. (2017), S. 151; Fischbach, D. (2003), S. 92 f./102; Fissenwert, P. (2012), S. 447; Quick, R./Höller, F./Koprivica, R. (2008), S. 25; Steller, M. (2011), S. 108.

814 Siehe AKEIÜ (Hrsg.) (2000), S. 2282 f.; Lück, W. (1999), S. 443; Pfitzer, N./Höreth, U. (2005), S. 170; Steller, M. (2011), S. 107.

815 Vgl. Albersmann, B. T./Hohenfels, D. (2017), S. 151; Fischbach, D. (2003), S. 206-215; Steller, M. (2011), S. 110 f. Allgemeiner Spindler, G. (2015a), § 107 AktG, Tz. 140. 
gen in der Anwendung von Rechnungslegungsgrundsätzen und internen Kontrollverfahren verfügen “"816 ${ }^{\text {sollen. }}{ }^{817}$

Ferner können Prüfungsausschüsse die Aufsichtsratsvorsitzenden entlasten, ${ }^{818}$ wenn zwischen den Aufsichtsratsvorsitzenden und den Vorsitzenden der Prüfungsausschüsse keine Personalunionen bestehen. Schließlich dürften ohne Prüfungsausschüsse oder bei Personalunionen zwischen den Aufsichtsratsvorsitzenden und den Vorsitzenden der Prüfungsausschüsse häufig die Aufsichtsratsvorsitzenden als Ansprechpartner für Abschlussprüfer und interne Revisoren dienen, ${ }^{819}$ obwohl sie möglicherweise im Bereich der Rechnungslegung nicht ausreichend qualifiziert sind. ${ }^{820}$ Die fehlenden Fachkenntnisse können dazu führen, dass Aufsichtsratsvorsitzende etwaige Hinweise der Abschlussprüfer oder der internen Revisoren auf abschlusspolitische Ergebnisspaltungen nicht angemessen berücksichtigen. ${ }^{821}$ Allerdings empfiehlt der DCGK erst ab dem Jahr 2012 keine Personalunionen zwischen Aufsichtsratsvorsitzenden und Vorsitzenden der Prüfungsausschüsse. ${ }^{822}$ Zuvor enthielt der DCGK lediglich eine diesbezügliche Anregung, ${ }^{823}$ auf deren Befolgung die Erklärungen zum DCGK i. S. v. § 161 Abs. 1 Satz 1 AktG (i. d. F. vom 19. Juli 2002; 22. Dezember 2011) nicht eingehen müssen. Somit ist ungewiss, ob in Prüfungsausschüssen vor dem Jahr 2012 Personalunionen zwischen den Aufsichtsratsvorsitzenden und den Vorsitzenden der Prüfungsausschüsse bestanden, weshalb dieses Kriterium in der empirischen Untersuchung unberücksichtigt bleibt.

\subsubsection{Zwischenfazit}

In den vorherigen Unterabschnitten wurde untersucht, ob Aufsichtsräte Vorstände von abschlusspolitischen Ergebnisspaltungen abhalten können. Als Zwischenfazit ist festzuhalten, dass diese Fähigkeit von den Qualifikationen der Aufsichtsräte und von Prüfungsausschüssen abhängt. Die Qualifikationen können die Aktionäre durch die Wahl von geeigneten Aufsichtsräten beeinflussen. Empirisch ergibt aber sich das Problem, dass sich die genauen Qualifikationen der Aufsichtsräte nicht zuverlässig quantifizieren lassen. Prüfungsausschüsse mit Finanzexperten als Vorsitzenden, die nicht mit den Aufsichtsratsvor-

816 DCGK (2008), Tz. 5.3.2 Satz 2. Gemäß Kremer, T. (2018), Tz. 1298 geht die Empfehlung somit über die gesetzliche Pflicht gemäß $\$ 100$ Abs. 5 AktG (i.d.F. vom 25. Mai 2009) hinaus, dass bei kapitalmarktorientierten Gesellschaften ,mindestens ein unabhängiges Mitglied des Aufsichtsrats über Sachverstand auf den Gebieten Rechnungslegung oder Abschlussprüfung verfügen“ muss.

817 Vgl. Albersmann, B. T./Hohenfels, D. (2017), S. 152; Böcking, H.-J./Dutzi, A./Müßig, A. (2004), S. 426/429 f./436; Wild, A. (2010), S. 516. Im Folgenden werden Personen, die diese Anforderungen erfüllen, vereinfachend als Finanzexperten bezeichnet.

818 Analog Kremer, T. (2018), Tz. 1288; Pfitzer, N./Höreth, U. (2005), S. 170; Steller, M. (2011), S. 108.

819 Umkehrschluss zu Mattheus, D. (2009), S. 572 f.

$820 \S 100 \mathrm{Abs}$. $5 \mathrm{AktG}$ (i.d.F. vom 25. Mai 2009) verlangt nicht, dass Aufsichtsratsvorsitzende, ,über Sachverstand auf den Gebieten Rechnungslegung oder Abschlussprüfung“ verfügen müssen.

821 Umkehrschluss zu Böcking, H.-J./Dutzi, A./Müßig, A. (2004), S. 426/431/436; Deckert, M. (1996), S. 991.

822 Siehe DCGK (2012), Tz. 5.2 Abs. 2 Satz 2.

823 Siehe DCGK (2008), Tz. 5.2 Abs. 2 Satz 2. 
sitzenden identisch sind, können abschlusspolitische Ergebnisspaltungen effizienter und effektiver als die Plenen überwachen. Allerdings lassen sich etwaige Personalunionen zwischen den Aufsichtsratsvorsitzenden und den Vorsitzenden der Prüfungsausschüsse empirisch nicht zuverlässig quantifizieren.

\subsubsection{Gesamtfazit und Formulierung der Hypothese}

Im vorliegenden Abschnitt wurde untersucht, ob Aufsichtsräte Vorstände von einer abschlusspolitischen Ergebnisspaltung abhalten wollen und können. Als Gesamtfazit ist festzuhalten, dass deren Wille mit fixen Vergütungen in der Praxis am besten gewährleistet werden kann, da die Haftungs- und Reputationsrisiken zumindest bei kapitalmarktorientierten Unternehmen ausreichend hoch sein dürften, damit sich die Aufsichtsräte trotz fixen Vergütungen ausreichend anstrengen. Die Fähigkeit von Aufsichtsräten, abschlusspolitische Ergebnisspaltungen zu erkennen, kann durch Prüfungsausschüsse mit Finanzexperten als Vorsitzenden erhöht werden. ${ }^{824}$ Folglich können Aufsichtsräte, die fix vergütet werden und über Prüfungsausschüsse mit Finanzexperten als Vorsitzenden verfügen, abschlusspolitische Ergebnisspaltungen tendenziell verhindern. Basierend auf diesen Erkenntnissen wird die folgende Alternativhypothese formuliert:

$\mathrm{H}_{2.1}$ : [Aufsichtsräte] Wenn für Aufsichtsräte ausschließlich fixe Vergütungssysteme und Prüfungsausschüsse mit Finanzexperten als Vorsitzenden existieren, verhindern sie abschlusspolitische Ergebnisspaltungen mit aufgegebenen Geschäftsbereichen.

Die zugehörige Nullhypothese, die abgelehnt werden muss, um die obige Alternativhypothese annehmen zu können, lautet:

$\mathrm{HO}_{2.1}$ : Wenn für Aufsichtsräte ausschließlich fixe Vergütungssysteme und Prüfungsausschüsse mit Finanzexperten als Vorsitzenden existieren, verhindern sie keine abschlusspolitischen Ergebnisspaltungen mit aufgegebenen Geschäftsbereichen.

\subsection{Anreizorientierte Vergütungssysteme für Vorstände}

\subsubsection{Zielsetzung}

Anreizorientierte Vergütungssysteme für Unternehmensleitungen wurden in der Tab. 5 als interne Corporate-Governance-Mechanismen klassifiziert. Anreizorientierte Vergütungssysteme sollen die Interessen der Aktionäre und Vorstände angleichen. ${ }^{825}$ Damit anreizorientierte Vergütungssysteme die Interessen angleichen können, müssen sie Anreize set-

824 Ungefähr auch Velte, P./Stiglbauer, M. (2011), S. 19.

825 Vgl. Ampenberger, M. (2010), S. 58; Steller, M. (2011), S. 24 f. Allgemein DeFond, M. L. (1992), S. 21. 
zen, damit die Vorstände die Unternehmen i. S. d. Aktionäre führen. ${ }^{826}$ Bezogen auf die Problemstellung in der vorliegenden Arbeit muss untersucht werden, ob anreizorientierte Vergütungssysteme verhindern, dass Vorstände abschlusspolitische Ergebnisspaltungen vornehmen wollen. Die ebenfalls relevante Frage nach der diesbezüglichen Fähigkeit ist leicht zu bejahen, da Vorstände für die Erstellung der Jahres- bzw. Konzernabschlüsse und somit für etwaige abschlusspolitische Ergebnisspaltungen verantwortlich sind ${ }^{827}$ Daher wird im Folgenden nur die Frage untersucht, ob Vorstände auf abschlusspolitische Ergebnisspaltungen verzichten wollen, wenn für sie geeignete anreizorientierte Vergütungssysteme existieren.

\subsubsection{Fixe und variable Vergütungen ohne Aktien und Aktienoptionen}

Würden Vorstände unter der alleinigen Maxime vergütet, dass abschlusspolitische Ergebnisspaltungen verhindert werden sollen, wären ausschließlich fixe Vergütungen anreizoptimal. ${ }^{828}$ Denn ausschließlich fixe Vergütungen entkoppeln Vorstände vom tatsächlichen oder wahrgenommenen Unternehmenserfolg, sodass Vorstände keine Anreize für abschlusspolitische Maßnahmen haben. ${ }^{829}$ Allerdings besteht das übergeordnete Ziel von anreizorientierten Vergütungssystemen nicht darin, Vorstände von abschlusspolitischen Maßnahmen abzuhalten. Vielmehr sind Anreize zu schaffen, damit sämtliche Unternehmensaufgaben i. S. d. Aktionäre erfüllt werden. ${ }^{830}$ Bei ausschließlich fix vergüten Vorständen ist dagegen zu befürchten, dass sie sich lediglich so sehr anstrengen, wie erforderlich ist, um nicht abberufen zu werden. ${ }^{831}$ Als Lösung für dieses Motivationsproblem kommen zunächst variable Vorstandsvergütungen ohne Aktien und Aktienoptionen, nachfolgend vereinfachend nur variable Vergütungen genannt, in Betracht. ${ }^{832}$ Bei variablen Vergütungen müssen Vorstandsleistungen jedoch beurteilt werden. Diese Leistungsbeurteilungen sind aufgrund von Hidden-Actions- und Hidden-Informations-Problemen typischerweise

826 Ähnlich Jensen, M. C./Murphy, K. J. (1990a), S. 242 f.; Stratmann, J. (2005), S. 152.

827 So $\$ 91 \mathrm{Abs}$. $1 \mathrm{AktG}$.

828 Vgl. Baiman, S. (1979), S. 27 f. Allgemeiner Datar, S./Alles, M. (1999), S. 28. Gemäß Dauner-Lieb, B. (2019a), § 87 AktG, Tz. 27 geht der Gesetzgeber aufgrund des Begriffs „Vergütungsstruktur“ im § $87 \mathrm{Abs}$. 1 Satz $2 \mathrm{AktG}$ ganz selbstverständlich davon aus, dass Vorstandsvergütungen nicht nur aus einer einzigen Komponente bestehen. Dennoch halten Dauner-Lieb, B. (2019a), § 87 AktG, Tz. 27; Fleischer, H. (2009), S. 803; Hoffmann-Becking, M./Krieger, G. (2009), S. 2; Hohenstatt, K.-S. (2009), S. 1351 ausschließlich fixe Vorstandsvergütungen für zulässig.

829 Siehe Antle, R. (1982), S. 512; Baiman, S. (1979), S. 27 f. Gemäß Holmström, B./Ricart i Costa, J. (1986), S. 836-838/856 f. werden fix vergütete Vorstände nur von der Sorge um ihre Reputationen geleitet.

830 Vgl. Bühner, R. (1989), S. 2181; Datar, S./Alles, M. (1999), S. 407; Dauner-Lieb, B. (2019a), §87 AktG, Tz. 26; Husemann, W. (1992), S. 55.

831 Grundlegend Ijiri, Y. (1971), S. 5/7 f./10; Ng, D. S./Stoeckenius, J. (1979), S. 8/15; Shavell, S. (1979), S. 56. Auch Antle, R. (1982), S. 512; Husemann, W. (1992), S. 35; Paulitschek, P. (2009), S. 55 f.; Spremann, K. (1988), S. 617.

832 Vgl. Francis, J. R./Wilson, E. R. (1988), S. 666; Palmrose, Z.-V. (1984), S. 234. 
schwierig. ${ }^{833}$ Zum einen hängt der Unternehmenserfolg nicht nur von den Anstrengungen der Vorstände, sondern auch von exogenen Risiken ab. ${ }^{834}$ Beispielsweise können Vorstände bei schlechter konjunktureller Lage trotz hohen Anstrengungen bestraft und bei guter konjunktureller Lage trotz niedrigen Anstrengungen belohnt werden. ${ }^{835}$ Aufgrund dieser exogenen Risiken kann es sinnvoll sein, die variable Vergütung an der Entwicklung des Aktienkurses des vom Vorstand geleiteten Unternehmens in Relation zur Entwicklung der Aktienkurse von ähnlichen Unternehmen derselben Branche zu bemessen. ${ }^{836}$ Zum anderen bergen variable Vergütungen die Gefahr, dass Vorstände die Parameter für die Leistungsbeurteilungen opportunistisch beeinflussen. ${ }^{837}$

Vor dem Hintergrund der Prinzipal-Agenten-Theorie, die von opportunistischen Vorständen ausgeht, ist abzuwägen, ob Vergütungssysteme Vorstände entweder zu einer unverzerrten Rechnungslegung und Anstrengungen entgegen dem Aktionärsinteresse oder zu einer verzerrten Rechnungslegung und Anstrengungen im Aktionärsinteresse motivieren sollen. ${ }^{838}$ Da diese beiden Extreme typischerweise nicht i. S.d. Aktionäre sein dürften, sind in der Praxis Vergütungssysteme zu erwarten, die fixe und variable Komponenten umfassen. ${ }^{839}$ Zudem entspricht die Kombination von fixen und variablen Vergütungen der Empfehlung des DCGK, die - trotz zahlreicher Änderungen bei den sonstigen Empfehlungen und Anregungen hinsichtlich Vorstandsvergütungen - im relevanten Zeitraum von 2008 bis 2013 bestand. ${ }^{840}$ Daher wird im Folgenden für verschiedene Arten von variablen Vergütungen untersucht, ob diese Vorstände zu hohen Anstrengungen bei gleichzeitigem Verzicht auf abschlusspolitische Ergebnisspaltungen motivieren können.

833 Sinngemäß Grossman, S. J./Hart, O. D. (1980), S. 48; Hart, O. D. (1983), S. 367; Holmström, B./Tirole, J. (1989), S. 89; Husemann, W. (1992), S. 57; Jensen, M. C./Murphy, K. J. (1990a), S. 226; Myers, S. C. (2001), S. 96.

834 Grundlegend Holmström, B. (1979), S. 81 f./88 f.; Shavell, S. (1979), S. 55. Sehr ähnlich Grossman, S. J./Hart, O. D. (1980), S. 48; Scharfstein, D. (1988b), S. 185 f. Sinngemäß Easterbrook, F. H./Fischel, D. R. (2001), S. 281; Jensen, M. C./Murphy, K. J. (1990a), S. 226; Jensen, M. C./Murphy, K. J. (1990b), S. 142; Jensen, M. C./Ruback, R. S. (1982), S. 30 f.

835 So Spremann, K. (1988), S. 615: „Fleiß und Pech können das gleiche bewirken wie Müßiggang in Verbindung mit Glück.“Auch Spremann, K. (1989), S. 743. Gemäß Bebchuk, L. A./Fried, J. M. (2004), S. 139 können fallende Zinsen die Aktienkurse von Unternehmen ohne Zutun der Vorstände erhöhen.

836 So The Royal Swedish Academy of Sciences (Hrsg.) (2016), S. 2 unter Bezugnahme auf die grundlegenden Erkenntnisse von Holmström, B. (1979), S. 89; Shavell, S. (1979), S. 56 f. Konkreter lässt sich diese Empfehlung allerdings aus Holmström, B. (1982b), S. 325/336 f. ableiten. Hierzu Ampenberger, M. (2010), S. 59 f.; Denis, D. K. (2001), S. 207 f.; Easterbrook, F. H./Fischel, D. R. (2001), S. 281.

837 Grundlegend Ijiri, Y. (1975), S. 35; Ng, D. S. (1978), S. 917-919; Ng, D. S./Stoeckenius, J. (1979), S. 1 f. Siehe auch Ampenberger, M. (2010), S. 59, Fn. 126; Binz, M./Sorg, M. (2002), S. 1274; Ewert, R. (1993), S. 726; Grimmeisen, F. P. (2016), S. 150; Husemann, W. (1992), S. 56/58; Jensen, M. C.Murphy, K. J. (1990a), S. 246; Nobach, K. (2006), S. 187; Pfleger, G. (1991), S. 25; Shleifer, A./Vishny, R. W. (1997), S. 745; Tirole, J. (2006), S. 22.

838 Abstrakt Ng, D. S./Stoeckenius, J. (1979), S. 5/8; Shavell, S. (1979), S. 56. Konkreter Paulitschek, P. (2009), S. 55 f.

839 Vgl. Mertens, H.-J./Cahn, A. (2009), § 87 AktG, Tz. 22; Werder, A. v./Turkali, J. (2015), S. 1358/1361.

840 Siehe DCGK (2002; 2013), Tz. 4.2.3 Satz 1. 
Variable Vergütungen, die sich am kurzfristigen Unternehmenserfolg bemessen, schaffen für Vorstände Anreize für abschlusspolitische Ergebnisspaltungen. ${ }^{841}$ Die Anreize für abschlusspolitische Ergebnisspaltung sind besonders groß, wenn sich die variablen Vergütungen an Ergebnissen aus den fortgeführten Geschäftsbereichen bemessen, da sich diese Ergebnisse durch abschlusspolitische Ergebnisspaltungen direkt erhöhen lassen. ${ }^{842}$ Indirekte Anreize für abschlusspolitische Ergebnisspaltungen existieren aber auch dann, wenn sich variable Vorstandsvergütungen an Marktgrößen, wie etwa Börsenkursen, bemessen. Reagieren die Kapitalmärkte positiv, weil nicht oder nicht vollständig erkannt wird, dass unerwartet hohe Ergebnisse aus den fortgeführten Geschäftsbereichen durch abschlusspolitische Ergebnisspaltungen herbeigeführt wurden, steigen auch variable Vorstandsvergütungen, die sich an Marktgrößen bemessen. ${ }^{843}$ Ebenso können Vorstände von positiven Kapitalmarktreaktionen profitieren, wenn sie ihre Aktien verkaufen oder ihre Aktienoptionen einlösen und die erhaltenen Aktien anschließend verkaufen, sofern für diese keine Wartezeiten respektive Haltefristen vorgegeben sind. ${ }^{844}$ Somit begünstigen variable Vergütungen mit kurzfristigen Bemessungsgrundlagen abschlusspolitische Ergebnisspaltungen, anstatt sie zu verhindern. Allerdings könnten sich Vorstände aufgrund von variablen Vergütungen mit kurzfristigen Bemessungsgrundlagen mehr anstrengen. ${ }^{845}$ Diese höheren Anstrengungen dürfte aber in den seltensten Fällen zugunsten der Aktionäre erfolgen. ${ }^{846}$ Vielmehr dürften opportunistische Vorstände Maßnahmen ergreifen, die ihren kurzfristigen Vergütungsinteressen zuträglich, aber den langfristigen Aktionärsinteressen abträglich sind. ${ }^{847}$ Beispielsweise könnten Vorstände Aufwendungen für Forschung und Entwicklung, Werbung oder Weiterbildungen kürzen, wodurch Unternehmen kurzfristig profitabler, aber langfristig typischerweise unprofitabler werden. ${ }^{848}$ Ebenso könnten Vorstände momentan defizitäre Geschäftsbereiche, die langfristig aber wieder profitabel werden dürften, aufgeben. ${ }^{849}$ Somit können variable Vergütungen mit kurzfristigen Bemessungsgrundlagen weder abschlusspolitische Ergebnisspaltungen noch Anstrengungen entgegen

841 Ebenso Binz, M./Sorg, M. (2002), S. 1274; Elschen, R. (2011), S. 600. Allgemeiner Bernhardt, W./Witt, P. (1997), S. 87 f.; Bühner, R. (1989), S. 2182; Friedrichsen, S. (2000), S. 25 f.; Healy, P. M. (1985), S. 89; Hoffmann-Becking, M./Krieger, G. (2009), S. 2; Mertens, H.-J./Cahn, A. (2009), § 87 AktG, Tz. 55; Quick, R. (1997), S. 726. Hoffmann-Becking, M./Krieger, G. (2009), S. 2 sehen in variablen Vergütungen mit kurzfristigen Bemessungsgrundlagen die Gefahr, ,,dass ein unternehmerisches ,Strohfeuer" belohnt wird."

842 Siehe analog für Aufsichtsräte den Abschnitt 6.1.

843 Vgl. Tirole, J. (2006), S. 19.

844 Hierzu Mertens, H.-J./Cahn, A. (2009), § 87 AktG, Tz. 37/66. In der vorliegenden Arbeit werden die Begriffe Wartezeit und Haltefrist wie bei Rieckers, O. (2019b), § 193 AktG, Tz. 32/34 unterschieden. Demnach handelt es sich bei Wartezeiten um die Dauer, bis Aktienoptionen ausgeübt werden dürfen. Dagegen handelt es sich bei Haltefristen um die Dauer, bis Aktien verkauft werden dürfen.

845 Vgl. Ampenberger, M. (2010), S. 59, Fn. 126.

846 Siehe Günzer, E. (2016), S. 15.

847 Vgl. Butler, H. N. (1989), S. 115; Tirole, J. (2006), S. 22. Dazu auch RegE VorstAG (2009), S. 5; Rechtsausschuss des Deutschen Bundestages (Hrsg.) (2009), S. 1.

848 Hierzu Almaleeh, N. M. S. (2019), S. 30; Fleischer, H. (2009), S. 802 f.; Friedrichsen, S. (2000), S. 25 f.; McVay, S. E. (2006), S. 524.

849 Siehe Friedrichsen, S. (2000), S. 26, Fn. 102. 
den Aktionärsinteressen verhindern. ${ }^{850} \mathrm{Ob}$ variable Vergütungen mit langfristigen Bemessungsgrundlagen diese Probleme lösen können, wird nachfolgend diskutiert.

Maßnahmen, die den Unternehmenserfolg kurzfristig erhöhen, schaden diesem oftmals langfristig. Vernachlässigen Vorstände bspw. Forschung und Entwicklung oder geben momentan defizitäre - aber strategisch wichtige - Geschäftsbereiche überhastet auf, büßen die betroffenen Unternehmen typischerweise Wettbewerbsvorteile ein, wodurch langfristige Unternehmenserfolge und somit auch variable Vergütungen mit langfristigen Bemessungsgrundlagen sinken. Folglich motivieren variable Vergütungen mit langfristigen Bemessungsgrundlagen Vorstände typischerweise zu Anstrengungen im Interesse der Aktionäre. Ob Vorstände variable Vergütungen mit langfristigen Bemessungsgrundlagen durch abschlusspolitische Ergebnisspaltungen beeinflussen können, hängt von der Art der verwendeten Aufwendungen und Erträge sowie von der Dauer bis zum Abgang von aufgegebenen Geschäftsbereichen ab. Verwenden Vorstände nur einmalig anfallende Aufwendungen und Erträge für abschlusspolitische Ergebnisspaltungen, können sie variable Vergütungen mit langfristigen Bemessungsgrundlagen nicht opportunistisch beeinflussen. Verwenden Vorstände dagegen regelmäßig anfallende Aufwendungen und Erträge für abschlusspolitische Ergebnisspaltungen, können sie damit variable Vergütungen mit langfristigen Bemessungsgrundlagen beeinflussen, bis die von ihnen geleiteten Unternehmen über keine aufgegebenen Geschäftsbereiche mehr verfügen. ${ }^{851}$ Beispielsweise können Vorstände bei hohen tatsächlichen Ergebnissen aus den fortgeführten mittels abschlusspolitischen Ergebnisspaltungen Cookie Jars anlegen, um mit diesen später bei niedrigen tatsächlichen Ergebnissen aus den fortgeführten Geschäftsbereichen Referenzwerte abschlusspolitisch zu erreichen. ${ }^{852}$ Analog zu variablen Vergütungen mit kurzfristigen Bemessungsgrundlagen existieren diese abschlusspolitischen Anreize sowohl, wenn Ergebnisse aus den fortgeführten Geschäftsbereichen die Bemessungsgrundlagen bilden, als auch in abgeschwächter Form, wenn Marktgrößen als Bemessungsgrundlagen dienen. Langfristig können Vorstände Ergebnis- und Marktgrößen mit abschlusspolitischen Ergebnisspaltungen aber nur schwer steuern, da die Voraussetzungen für den Ausweis von aufgegebenen Geschäftsbereichen restriktiv sind. ${ }^{853}$ Somit sind die Anreize für Vorstände, auf abschlusspolitische Ergebnisspaltungen zu verzichten und i.S. d. Aktionäre zu arbeiten, bei variablen Vergütungen mit langfristigen Bemessungsgrundlagen deutlich höher als bei kurzfristigen Bemessungsgrundlagen. ${ }^{854} \mathrm{Da}$ als variable Vergütungen mit langfristigen Bemessungsgrund-

850 Anders vermutlich Jensen, M. C./Murphy, K. J. (1990b), S. 145.

851 Siehe den Unterabschnitt 4.3.3.

852 Siehe zum Erreichen von Referenzwerten den Unterabschnitt 5.4.1 und zum Anlegen von Cookie Jars den Unterabschnitt 5.6.1.

853 Siehe den Unterabschnitt 4.1.1 und den Abschnitt 11.7.

854 Analog Friedrichsen, S. (2000), S. 29. 
lagen häufig Aktien und Kaufoptionen auf Aktien verwendet werden, ${ }^{855}$ soll nachfolgend untersucht werden, ob diese für Vorstände Anreize setzen, auf abschlusspolitische Ergebnisspaltungen zu verzichten und i. S. d. Aktionäre zu agieren.

\subsubsection{Variable Vergütungen mit Aktien und Aktienoptionen}

Im Jahr 2009 wurde durch das Gesetz zur Angemessenheit der Vorstandsvergütung zum einen die Pflicht, dass die „Vergütungsstruktur [...] bei börsennotierten Gesellschaften auf eine nachhaltige Unternehmensentwicklung auszurichten [ist] ${ }^{1656}$, und zum anderen die Empfehlung, dass ,[v]ariable Vergütungsbestandteile [...] daher eine mehrjährige Bemessungsgrundlage haben [sollen] ${ }^{* 857}$ in das Aktiengesetz aufgenommen. ${ }^{858}$ Die mehrjährige Bemessungsgrundlage für variable Vergütungsbestandteile wurde dahingehend konkretisiert, dass Beschlüsse über Bezugsrechte für die Mitglieder von Geschäftsführungen, die in nach dem 5. August 2009 einberufenen Hauptversammlungen gefasst wurden, ${ }^{859}$ Wartezeiten für erstmalige Ausübungen in Höhe von nunmehr mindestens vier ${ }^{860}$ anstatt, wie zuvor, mindestens zwei Jahre ${ }^{861}$ vorsehen müssen. ${ }^{862}$ Somit ging der Gesetzgeber bei Bezugsrechten, die häufig als Grundlage von Aktienoptionsprogrammen dienen, ${ }^{863}$ über die damalige Anregung des DCGK, dass die variablen Vergütungsteile ,auch Komponenten mit langfristiger Anreizwirkung und Risikocharakter enthalten [sollten] ${ }^{\star 864}$, hinaus. Für Vorstandsvergütungen mit Aktien wurde keine gesetzliche Pflicht zu einer bestimmten Haltefrist eingeführt, ${ }^{865}$ obwohl sie ohne angemessene Haltefristen die gleichen Fehlanrei-

855 Siehe RegE VorstAG (2009), S. 5. Ebenso bereits Butler, H. N. (1989), S. 116; Friedrichsen, S. (2000), S. 13-15; Shleifer, A./Vishny, R. W. (1997), S. 744. Mit Kaufoptionen auf Aktien - nachfolgend vereinfachend Aktienoptionen genannt - erwerben deren Inhaber das Recht, aber nicht die Pflicht, Aktien zu einem bestimmten Zeitpunkt oder in einem bestimmten Zeitraum zu einem bestimmten Ausübungspreis zu kaufen; vgl. Grottel, B. (2020a), § 285 HGB, Tz. 253. Zum Ausübungszeitpunkt sind Aktienoptionen nur dann wertvoll, wenn die Ausübungspreise unter dem Marktpreis der Aktien liegen; vgl. Tirole, J. (2006), S. 23. Dann können die ehemaligen Inhaber der Optionen durch sofortige Verkäufe der Aktien Gewinne realisieren, die sich aus den Differenzen zwischen dem Marktpreis der Aktien und den Ausübungspreisen der Optionen ergeben; vgl. Friedrichsen, S. (2000), S. 181 f. Der kleinste Wert, den Optionen annehmen können, ist Null, da Optionsinhaber die Aktien nicht kaufen müssen, wenn der Marktpreis der Aktien unter den Ausübungspreisen der Optionen liegen.

$856 \S 87$ Abs. 1 Satz 2 AktG (i.d.F. vom 31. Juli 2009).

$857 \S 87$ Abs. 1 Satz 3 AktG.

858 Siehe Art. 1 Nr. 1 Buchst. a) VorstAG.

859 Siehe Art. 2 VorstAG.

860 So $\$ 193$ Abs. $2 \mathrm{Nr} .4 \mathrm{AktG}$.

861 Siehe $\$ 193$ Abs. 2 Nr. 4 AktG (i.d.F. vom 27. April 1998).

862 Siehe Art. 1 Nr. 1 Buchst. a) VorstAG; RegE VorstAG (2009), S. 5. Dazu Dauner-Lieb, B. (2019a), $\S 87$ AktG, Tz. 22.

863 Vgl. Bernhardt, W./Witt, P. (1997), S. 86; Fuchs, A. (2016), § 193 AktG, Tz. 23; Hermanns, M. (2019), $\S 193$ AktG, Tz. 9; Poelzig, D. (2013a), § 285 HGB, Tz. 171.

864 DCGK (2008), Tz. 4.2.3 Abs. 2 Satz 2.

865 Hierzu Fuchs, A. (2016), § 193 AktG, Tz. 33; Hermanns, M. (2019), § 193 AktG, Tz. 9; Mertens, H. J./Cahn, A. (2009), § 87 AktG, Tz. 66; Rieckers, O. (2019b), § 193 AktG, Tz. 34. Gemäß Fuchs, A. (2016), § 193 AktG, Tz. 33; Hermanns, M. (2019), § 193 AktG, Tz. 9; Rieckers, O. (2019b), $\S 193$ AktG, Tz. 34 sind Haltefristen für Aktien, die Vorstände als Vergütungen erhalten, in der Praxis üblich. Anders Friedrichsen, S. (2000), S. 181 f.; Schmid in Wadewitz, S. (2016), S. 9. 
ze wie variable Vergütungen mit kurzfristigen Bemessungsgrundlagen setzen können. ${ }^{866}$ Nachfolgend werden wesentliche Vor- und Nachteile von Vorstandsvergütungen mit Aktien und Aktienoptionen aus Sicht der Prinzipal-Agenten-Theorie beleuchtet. Dabei wird angenommen, dass neben der gesetzlichen Pflicht zu mindestens vierjährigen Wartezeiten für Aktienoptionen auch für Aktien angemessene Haltefristen vereinbart wurden. ${ }^{867}$

Werden Vorständen Aktien oder Aktienoptionen gewährt, haben diese Anreize, den Marktpreis der Aktien zu erhöhen, um somit die Werte ihrer Aktien oder Aktienoptionen zu erhöhen. ${ }^{868}$ Da Aktionäre ebenfalls an Werterhöhungen ihrer Aktien interessiert sind, ist der zentrale Vorteil von Aktien und Aktienoptionen, dass sie die Interessen der Vorstände und Aktionäre angleichen. ${ }^{869}$ Der wesentliche Unterschied zwischen Aktien und Aktienoptionen ist, dass Aktien nur dann wertlos sind, wenn deren Marktpreise gleich null sind, während Aktienoptionen auch dann wertlos sein können, wenn der Marktpreis der Aktien zum Ausübungszeitpunkt zwar größer als null ist, aber die Ausübungspreise der Optionen über dem Marktpreis der Aktien liegen. Aus Sicht der Aktionäre erscheinen Vorstandsvergütungen mit Aktienoptionen somit grundsätzlich als vorteilhafter als Vorstandsvergütungen mit Aktien, da erfolglose Vorstände mit wertlosen Aktienoptionen stärker als mit wertgeminderten Aktien sanktioniert werden. Allerdings kann sich dieser Aspekt auch zum Nachteil der Aktionäre entwickeln, wenn Vorstände über eine starke Verhandlungsmacht verfügen und aufgrund von nahezu wertlosen Aktienoptionen Nachverhandlungen fordern. ${ }^{870}$ Ebenso zwiespältig sind die aus Aktienoptionen resultierenden Anreize für Vorstände, Risiken einzugehen, um den Marktpreis der Aktien über die Ausübungspreise der Aktienoptionen zu treiben. ${ }^{871}$ Solange Aktienoptionen risikoaverse Vorstände motivieren, angemessene Risiken einzugehen, sind Vorstandsvergütung mit Aktienoptionen für Aktionäre vorteilhaft. ${ }^{872}$ Sobald Aktienoptionen Vorstände jedoch zu unangemessenen Risiken verleiten, sind sie für Aktionäre nachteilig. ${ }^{873}$ Dann können Vergütungen mit Aktien die bessere Wahl sein, da sie Vorstände ebenso wie Aktionäre an Kursverlusten partizipieren lässt. ${ }^{874}$ Unabhängig davon, ob Vorstände unmittelbar mit Aktien vergütet werden oder

866 Weiterführend Bebchuk, L. A./Fried, J. M. (2003), S. 85-87. Siehe auch den Abschnitt 6.2.

867 Siehe auch Bernhardt, W./Witt, P. (1997), S. 98.

868 Siehe Bernhardt, W./Witt, P. (1997), S. 86/89; Easterbrook, F. H./Fischel, D. R. (2001), S. 282; Friedrichsen, $S$. (2000), S. 13-15.

869 Grundlegend Jensen, M. C./Meckling, W. H. (1976), S. 312 f. Hierzu auch Bernhardt, W./Witt, P. (1997), S. 91/97; Bress, S. (2008), S. 46; DeFond, M. L. (1992), S. 21; Denis, D. K. (2001), S. 203; Feser; N. (2009), S. 15; Friedrichsen, S. (2000), S. 27; Fuchs, A. (2016), § 193 AktG, Tz. 23; Jensen, M. C./Warner, J. B. (1988), S. 5/7; Köke, J. (2004), S. 57; Rieckers, O. (2019b), § 193 AktG, Tz. 23.

870 Siehe Bebchuk, L. A./Fried, J. M. (2004), S. 127; Tirole, J. (2006), S. 24. Dazu auch Bernhardt, W./Witt, P. (1997), S. 89 f.; Denis, D. K. (2001), S. 207 f.; Ronen, J./Yaari, V. (2008), S. 12.

871 Vgl. Bühner, R. (1989), S. 2183; Friedrichsen, S. (2000), S. 15; Tirole, J. (2006), S. 23 f.

872 Vgl. Friedrichsen, S. (2000), S. 27.

873 So Mertens, H.-J./Cahn, A. (2009), § 87 AktG, Tz. 56. Gemäß Denis, D. K. (2001), S. 201 f. leiten Vorstände Unternehmen tendenziell risikoavers, da ein Großteil ihres Humankapitals typischerweise in den Unternehmen gebunden ist und die Wertentwicklung des Humankapitals von der Unternehmensentwicklung abhängt.

874 Dazu Bebchuk, L. A./Fried, J. M. (2004), S. 139 f.; Bernhardt, W./Witt, P. (1997), S. 90/97; Friedrichsen, S. (2000), S. 15; Monks, R. A. G. (1995), S. 230. 
diese mittelbar durch ausgeübte Aktienoptionen erlangen, ${ }^{875}$ löst ein Aktienbesitz der Vorstände nicht sämtliche Prinzipal-Agenten-Probleme zwischen den restlichen Aktionären und den Vorständen. Insbesondere verbleiben zwei Probleme.

Erstens bestehen für opportunistische Vorstände, welche die von ihnen geleiteten Unternehmen nicht vollständig innehaben, Anreize zu Verhaltensweisen und Transaktionen, die ihnen nutzen, aber den restlichen Aktionären schaden. ${ }^{876}$ Verkauft ein Vorstand bspw. aus seinem Privateigentum ein Grundstück zu einem Preis, der 1.000 GE über dem Marktwert liegt, an das von ihm geleitete Unternehmen, ist diese Transaktion für den Vorstand auch dann noch vorteilhaft, wenn ihm 60 Prozent der Aktien des Unternehmens gehören. ${ }^{877} \mathrm{Ob}$ wohl der Wert der Vorstandsaktien durch die Transaktion c. p. um 0,6 $1.000 \mathrm{GE}=600 \mathrm{GE}$ sinkt, ${ }^{878}$ ist die Transaktion für den Vorstand vorteilhaft, da sein privater Ertrag aus dem Grundstücksverkauf in Höhe von 1.000 GE den privaten Aufwand aus dem Wertverlust der Aktien in Höhe 600 GE übersteigt, sodass dem Vorstand ein privater Gewinn in Höhe von $1.000 \mathrm{GE}-600 \mathrm{GE}=400 \mathrm{GE}$ verbleibt. ${ }^{879} \mathrm{Im}$ Beispiel stellen die $400 \mathrm{GE}$ die Agency Costs dar, welche die restlichen Aktionäre durch den Wertverlust ihrer Aktien als Residual Loss zu tragen haben. Basierend auf diesen Erkenntnissen hat die Prinzipal-Agenten-Theorie die „Convergence-Of-Interests Hypotheses“ ${ }^{\star 880}$ hervorgebracht, die besagt, dass mit steigendem Aktienbesitz der Vorstände die Agency Costs sinken und die Unternehmenswerte steigen. ${ }^{881}$ Gemäß der Convergence-Of-Interests Hypotheses maximieren Vorstände Unternehmenswerte nur dann, wenn sie Alleineigentümer der Unternehmen sind und somit keine anderen Aktionäre übervorteilen können. ${ }^{882}$

Zweitens kann nicht pauschal unterstellt werden, dass mit dem Aktienbesitz der Vorstände deren Anreize, die restlichen Aktionäre zu übervorteilen, stetig und linear abnehmen. ${ }^{883}$ Vielmehr können Vorstände gemäß der „Entrenchment Hypotheses“ ${ }^{\text {“84 }}$ aufgrund ihres Aktienbesitzes schwerer zu überwachen und zu disziplinieren sein, ${ }^{885}$ wodurch die Agency

875 Gemäß Müller, W. (2018), § 1 AktG, Tz. 88 erlaubt es die für Kapitalgesellschaften charakteristische Fremdorganschaft durchaus, dass Organmitglieder auch Aktionäre sind und umgekehrt.

876 Vgl. Jensen, M. C./Meckling, W. H. (1976), S. 346. Ähnlich auch Denis, D. K. (2001), S. 193; Husemann, W. (1992), S. 53; La Porta, R. et al. (2000), S. 5; Palmrose, Z.-V. (1984), S. 232.

877 Siehe zu diesem Beispiel bereits Berle jr., A. A./Means, G. C. (1932), S. 122. Rechtliche Einwände gegen diese Transaktion sollen unberücksichtigt bleiben.

878 Analog Jensen, M. C./Meckling, W. H. (1976), S. 315; Paulitschek, P. (2009), S. 46.

879 Vgl. Berle jr., A. A./Means, G. C. (1932), S. 122. Allgemein hierzu La Porta, R. et al. (2000), S. 4.

880 Morck, R./Shleifer, A./Vishny, R. W. (1988), S. 293. Auch Bress, S. (2008), S. 46.

881 Vgl. Jensen, M. C./Meckling, W. H. (1976), S. 306/346; Kaserer, C./Moldenhauer, B. (2008), S. 2.

882 Anders Demsetz, H. (1983), S. 382 f., der auf ideelle Motive verweist. Ferner existieren in Unternehmen, die sich vollständig im Vorstandseigentum befinden, aufgrund der Personalunion zwischen den Aktionären und Vorständen keine Prinzipal-Agenten-Probleme.

883 Vgl. Kaserer, C./Moldenhauer, B. (2008), S. 2. Undifferenziert Jensen, M. C./Murphy, K. J. (1990b), S. 141.

884 Morck, R./Shleifer, A./Vishny, R. W. (1988), S. 294. Auch Bress, S. (2008), S. 46.

885 Vgl. Demsetz, H. (1983), S. 390; Denis, D. K. (2001), S. 203. Analog Fama, E. F./Jensen, M. C. (1983a), S. 314 f. Hierzu auch Shleifer, A./Vishny, R. W. (1997), S. 742 f. 
Costs steigen und die Unternehmenswerte sinken ${ }^{886}$ Beispielsweise können Vorstände, die über Aktien der von ihm geleiteten Unternehmen verfügen, ihnen wohlgesonnene Kandidaten als Aufsichtsräte vorschlagen ${ }^{887}$ und für diese stimmen. ${ }^{888}$ Werden derartige Kandidaten bestellt, ${ }^{889}$ erschweren sie die Überwachung und Disziplinierung der Vorstände durch die Aufsichtsräte. ${ }^{890}$ Ebenso können besondere Umstände wie langjährige Unternehmenszugehörigkeiten oder Stati als Unternehmensgründer, die häufig mit einem Aktienbesitz der Vorstände verbunden sind, deren Überwachung und Disziplinierung erschweren. ${ }^{891}$

Die Entrenchment Hypotheses widerspricht der Convergence-Of-Interests Hypotheses nicht zwingend. ${ }^{892}$ Vielmehr kann es von der Höhe des Aktienbesitzes abhängen, ob Vorstände im Interesse der restlichen Aktionäre handeln oder davon abweichende Interessen verfolgen. ${ }^{893} \mathrm{Da}$ Vorstände für Beschlüsse zu ihren Gunsten bestimmte Mehrheiten benötigen, ${ }^{894}$ könnte ein relativ niedriger Aktienbesitz der Vorstände deren Interessen tendenziell an die Interessen der restlichen Aktionäre i. S. d. Convergence-Of-Interests Hypotheses angleichen. Dagegen können Vorstände mit relativem hohen Aktienbesitz die erforderliche Mehrheiten erreichen und i.S.d. Entrenchment Hypotheses Beschlüsse zu Lasten der restlichen Aktionäre durchsetzen. ${ }^{895}$ Unabhängig davon, ob ein Aktienbesitz der Vorstände mit der Convergence-Of-Interests Hypotheses, der Entrenchment Hypotheses oder der soeben beschriebenen Kombination der beiden Hypothesen im Einklang steht, existieren hinsichtlich der empirischen Untersuchung des Aktienbesitzes der Vorstände in der vorliegenden Arbeit zwei Probleme.

Zum einen besteht im relevanten Zeitraum von 2008 bis 2013 keine gesetzliche Pflicht zur Angabe der Höhe des Aktienbesitzes der Vorstände, sondern es existieren lediglich diesbezügliche Empfehlungen im DCGK. ${ }^{896}$ Allerdings mussten sämtliche Aktionäre einschließlich etwaiger Organmitglieder die BaFin und den Emittenten informieren, wenn ihre Stimmrechte die Schwellenwerte in Höhe von drei, fünf, zehn, 15, 20, 25, 30, 50 oder

886 Siehe Morck, R./Shleifer, A./Vishny, R. W. (1988), S. 294. Hierzu auch Bress, S. (2008), S. 46.

887 Siehe zum Vorschlagsrecht $\S 127$ Abs. 1 Satz 1 AktG und zum Vorschlag von wohlgesonnene Kandidaten Bebchuk, L. A./Fried, J. M. (2004), S. 25 f.

888 Gemäß Koch, J. (2016c), § 136 AktG, Tz. $3 / 17$ f.; Liebscher, T. (2019b), § 136 AktG, Tz. 1; Rieckers, O. (2019a), § $136 \mathrm{AktG}, \mathrm{Tz} .1$ f./15 können Vorstände die Stimmrechte aus ihren Aktien bei der Wahl von Aufsichtsräten ausüben, da $\S 136 \mathrm{Abs}$. $1 \mathrm{Satz} 1 \mathrm{AktG}$ als kasuistische Ausschlussnorm Vorständen die Ausübung von Stimmrechten nicht verbietet.

889 Siehe $\$ 101$ Abs. 1 Satz 1 AktG.

890 Vgl. Becht, M./Bolton, P./Röell, A. (2003), S. 31.

891 Siehe Morck, R./Shleifer, A./Vishny, R. W. (1988), S. 294/312.

892 Vgl. Bress, S. (2008), S. 46, Fn. 8.

893 Hierzu Morck, R./Shleifer, A./Vishny, R. W. (1988), S. 311 f. Ähnlich Denis, D. K. (2001), S. 203.

894 Gemäß $\$ 133$ Abs. 1 Akt $G$ erfordern Beschlüsse der Hauptversammlung grundsätzlich die Mehrheit der abgegebenen Stimmen, wobei Satzungen gemäß $\S 133 \mathrm{Abs}$. $2 \mathrm{AktG}$ für Wahlen andere Bestimmungen treffen können.

895 Dazu Morck, R./Shleifer, A./Vishny, R. W. (1988), S. 311 f., die auch alternative Gründe für eine derartige Korrelation zwischen Unternehmenswerten und Vorstandsaktien diskutieren. Ähnlich Denis, D. K. (2001), S. 203.

896 Siehe DCGK (2008; 2009; 2012; 2013), Tz. 4.2.5. 
75 Prozent aller Stimmrechte erreichten, überschritten oder unterschritten. ${ }^{897}$ Speziell Personen mit Führungsaufgaben, zu denen Vorstände zählen, ${ }^{898}$ mussten oberhalb einer Bagatellgrenze in Höhe von 5.000 Euro pro Kalenderjahr ${ }^{899}$ der BaFin und dem Emittenten sogar sämtliche Geschäfte im Zusammenhang mit den Aktien des Arbeitgebers mitteilen. ${ }^{900}$ Diese beiden Mitteilungspflichten verpflichteten wiederum die Emittenten, die erhaltenen Informationen u. a. im Unternehmensregister zu veröffentlichen, ${ }^{901}$ das seit dem 1. Januar $2007^{902}$ elektronisch geführt wird. ${ }^{903}$ Allerdings sind im elektronischen Unternehmensregister Veröffentlichungen zum Aktienbesitz von Vorständen nur dann auffindbar, wenn sich dieser seit dem 1. Januar 2007 verändert hat. Verfügten Vorstände bspw. bereits im Jahr 2006 über Aktien des Emittenten und führten während des relevanten Zeitraums von 2008 bis 2013 keine privaten Transaktionen mit Aktien des Emittenten oberhalb der Bagatellgrenze durch, lässt sich der Aktienbesitz dem elektronischen Unternehmensregister nicht entnehmen. Hinsichtlich der empirischen Untersuchung in der vorliegenden Arbeit resultiert daraus das Problem, dass sich nicht abschätzen lässt, ob Vorstandsvergütungen mit Aktien entsprechend der Entrenchment Hypotheses oder der Convergence-Of-Interests Hypotheses wirken, da hierfür die Höhe des Aktienbesitzes bekannt sein muss. ${ }^{904}$

Zum anderen lässt sich die Höhe der Vorstandsvergütungen mit Aktien und Aktienoptionen nicht immer zuverlässig ermitteln. Zwar müssen Bezugsrechte und sonstige aktienbasierte Vergütungen mit ihrer Anzahl und den beizulegenden Zeitwerten zum Zeitpunkt ihrer Gewährung in Jahres- und Konzernabschlüssen separat angegeben werden und in den ausgewiesenen Gesamtvergütungen enthalten sein. ${ }^{905}$ Spätere Wertänderungen sind aber nur separat anzugeben und in den Gesamtvergütungen zu berücksichtigen, wenn sie auf veränderten Ausübungsbedingungen, wie etwa gesenkten Ausübungspreisen, ${ }^{906}$ nicht aber auf sonstigen Veränderungen, wie etwa schwankenden Aktienkursen, ${ }^{907}$ beruhen..${ }^{908}$ Somit sind Wertänderungen von Vergütungen mit Aktien oder Aktienoptionen, die daraus resultieren, dass Vorstände die Aktienkurse erhöhen, in den Geschäftsjahren nach deren

897 So § 21 Abs. 1 Satz 1 WpHG (i.d.F. vom 22. April 2002).

898 Siehe $\$ 15 a$ Abs. 2 WpHG (i.d. F. vom 16. Juli 2007).

899 Siehe $\$ 15 a$ Abs. 1 Satz 5 WpHG (i.d.F. vom 16. Juli 2007). Kritische Würdigung bei Riedl, A. M. (2008), S. 57-59.

900 So $\$ 15 a$ Abs. 1 Satz 1 WpHG (i.d.F. vom 16. Juli 2007).

901 So $\$ 26$ Abs. 1 Satz 1 WpHG (i.d.F. vom 5. Januar 2007); 26 Abs. 1 Satz 1 WpHG (i.d. F. vom 5. April 2011) hinsichtlich der Mitteilungspflicht bei Schwellenwerten und $\$ 15 a \mathrm{Abs} .4$ Satz $1 \mathrm{WpHG}$ (i.d.F. vom 16. Juli 2007) hinsichtlich der Mitteilungspflicht bei Geschäften von Vorständen.

902 Siehe Art. 13 Abs. 2 EHUG.

903 So $\S 8$ b Abs. 1 HGB (i.d.F. vom 10. November 2006; 31. August 2015).

904 Allgemeiner Jensen, M. C./Murphy, K. J. (1990b), S. 141.

905 So $\$ 285$ Nr. 9 Buchst. a) Satz 1/4 Hs. 1 HGB für Jahres- und $\S 314$ Abs. 1 Nr. 6 Buchst. a) Satz 1/4 Hs. 1 HGB für Konzernabschlüsse. Dazu Hennke, P./Fett, T. (2007), S. 1269 f.

906 So GesE VorstOG (2005), S. 7. Dazu Poelzig, D. (2013a), § 285 HGB, Tz. 174; Poelzig, D. (2013b), $\S 314$ HGB, Tz. 47.

907 Vgl. Grottel, B. (2020a), § 285 HGB, Tz. 253-255; Grottel, B. (2020b), §314 HGB, Tz. 95; Hohenstatt, K.-S./Wagner, T. (2008), S. 947.

908 Siehe $\S 285$ Nr. 9 Buchst. a) Satz 1/4 Hs. 2 HGB für Jahres- sowie $\S 314$ Abs. 1 Nr. 6 Buchst. a) Satz 1/4 Hs. 2 HGB und DRS 17.32 (2010) für Konzernabschlüsse. Hierzu Denis, D. K. (2001), S. 202. 
Gewährung nicht zuverlässig ermittelbar. ${ }^{909}$ Zugespitzt formuliert werden gerade diejenigen Teile der variablen Vergütungen, die für Vorstände typischerweise besonders lukrativ sind und die stärksten Leistungsanreize auslösen, nicht im Jahres- bzw. Konzernabschluss ausgewiesen. ${ }^{910}$ Hinsichtlich der empirischen Untersuchung in der vorliegenden Arbeit resultiert daraus das Problem, dass sich die Leistungsanreize aus Vergütungen mit Aktien und Aktienoptionen nicht zuverlässig abschätzen lassen.

\subsubsection{Fazit und Formulierung der Hypothese}

Im vorliegenden Abschnitt wurde untersucht, ob Vorstände aufgrund bestimmter Vergütungssysteme auf abschlusspolitische Ergebnisspaltungen verzichten wollen. Als Fazit ist festzuhalten, dass ausschließlich fixe Vergütungen hierzu am besten geeignet sind. Sollen sich Vorstände dagegen sowohl im Aktionärsinteresse anstrengen als auch weitgehend auf abschlusspolitische Ergebnisspaltungen verzichten, eignen sich hierzu variable Vergütungen mit langfristigen Bemessungsgrundlagen. Folglich resultieren aus fixen und variablen Vergütungen mit langfristigen Bemessungsgrundlagen für Vorstände Anreize, auf abschlusspolitische Ergebnisspaltungen zu verzichten. Hinsichtlich variablen Vergütungen mit langfristigen Bemessungsgrundlagen wird in der vorliegenden Arbeit nicht unterschieden, ob sie Aktien bzw. Aktienoptionen umfassen oder nicht, da sich deren mögliche Vorteile empirisch nicht zuverlässig erfassen lassen. Basierend auf diesen Erkenntnissen wird die folgende Alternativhypothese formuliert:

$\mathrm{H}_{2.2}$ : [Vorstandsvergütungen] Je höher die fixen und variablen Vergütungen mit langfristigen Bemessungsgrundlagen relativ zu den gesamten Vorstandsvergütungen, desto schwächer sind abschlusspolitische Ergebnisspaltungen mit aufgegebenen Geschäftsbereichen.

Die zugehörige Nullhypothese, die abgelehnt werden muss, um die obige Alternativhypothese annehmen zu können, lautet:

$\mathrm{H}_{2.2}$ : Je höher die fixen und variablen Vergütungen mit langfristigen Bemessungsgrundlagen relativ zu den gesamten Vorstandsvergütungen, desto stärker sind abschlusspolitische Ergebnisspaltungen mit aufgegebenen Geschäftsbereichen bzw. es gibt keinen diesbezüglichen Zusammenhang.

909 Vgl. Denis, D. K. (2001), S. 202; Hohenstatt, K.-S./Wagner, T. (2008), S. 947; Thüsing, G. (2005), S. 1392.

910 Kritisch bereits Binz, M./Sorg, M. (2002), S. 1275-1277. Hennke, P./Fett, T. (2007), S. 1269 gelangen nach historischer, systematischer und teleologischer Auslegung von $\$ \S 285$ Nr. 9 Buchst. a) Satz 1/4 Hs. 1; 314 Abs. 1 Nr. 6 Buchst. a) Satz 1/4 Hs. 1 HGB zu dem Ergebnis, dass Aktienoptionen nur in den Geschäftsjahren ihrer Gewährung in Jahres- respektive Konzernabschlüssen berichtet werden müssen. Thüsing, G. (2005), S. 1392 schreibt: „Das ist ein Fehler des Gesetzes.“ 


\subsection{Eigentümerstrukturen}

\subsubsection{Zielsetzung}

Eigentümerstrukturen wurden in der Tab. 5 als interne Corporate-Governance-Mechanismen klassifiziert, wobei geeignete Eigentümerstrukturen typischerweise mit hohen Beteiligungsquoten mindestens eines Aktionärs gleichgesetzt werden. ${ }^{911}$ Diese Großaktionäre sollen insbesondere die Informationsasymmetrien zwischen Aktionären und den Vorständen abbauen. ${ }^{912}$ Dahinter steht das Kalkül, dass die Monitoring Expenditures bei Großaktionären in günstigeren Verhältnissen zu deren potenziellen Nutzen aus dem Aktienbesitz in Form von Kurssteigerungen und Dividenden stehen als bei Kleinaktionären. ${ }^{913}$ Die günstigeren Kosten-Nutzen-Verhältnisse lassen eine genauere Vorstandsüberwachung durch Großaktionäre vermuten, von der auch Kleinaktionäre profitieren können. ${ }^{914} \mathrm{Be}$ zogen auf die Problemstellung in der vorliegenden Arbeit muss untersucht werden, ob Großaktionäre Vorstände von abschlusspolitischen Ergebnisspaltungen abhalten wollen und können.

\subsubsection{Mehrstufige Prinzipal-Agenten-Probleme}

Im Unterabschnitt 6.1.2 wurde aufgezeigt, dass zweistufige Prinzipal-Agenten-Probleme entstehen, wenn sich Aktionäre bei der Überwachung der Vorstände durch Aufsichtsräte vertreten lassen. Konkret bestehen die Probleme darin, dass Aufsichtsräte einerseits als Prinzipale die Vorstände überwachen sollen, andererseits aber Agenten der Aktionäre sind, die anstelle einer angemessenen Überwachung der Vorstände Eigeninteressen verfolgen können. Beeinflussen Großaktionäre die Vorstände unmittelbar, können analoge zweistufige Prinzipal-Agenten-Probleme auftreten, bei denen Großaktionäre einerseits als Prinzipale der Vorstände und andererseits als Agenten der Kleinaktionäre auftreten. ${ }^{915}$ Beeinflussen Großaktionäre Vorstände dagegen mittelbar über die Aufsichtsräte, entstehen sogar dreistufige Prinzipal-Agenten-Probleme, bei denen Großaktionäre einerseits als Prinzipale der Aufsichtsräte und andererseits als Agenten der Kleinaktionäre agieren, während die Aufsichtsräte wiederum einerseits als Prinzipale der Vorstände und andererseits als Agenten der Großaktionäre auftreten. Aufgrund dieser mehrstufigen Prinzipal-Agenten-Probleme

911 Die Vor- und Nachteile, wenn Vorstände Aktien der von ihnen geleiteten Unternehmen besitzen, wurden bereits im Unterabschnitt 6.2.3 diskutiert.

912 Vgl. Engelen, C. (2015), S. 934 f. Allgemeiner Köke, J. (2004), S. 55.

913 Nahezu identisch Ruhnke, K./Simons, D. (2018), S. 76. Ungefähr Ampenberger, M. (2010), S. 73; Bress, S. (2008), S. 47; Köke, J. (2004), S. 55; Stratmann, J. (2005), S. 159 f.

914 Vgl. Bassen, A. (2002), S. 93 f.; Engelen, C. (2015), S. 934 f.; Mello, A. S./Parsons, J. E. (1998), S. 103; Moldenhauer, B. (2007), S. 77 f.; Stratmann, J. (2005), S. 160. Im Ergebnis auch Tirole, J. (2006), S. 20.

915 Ungefähr Bassen, A. (2002), S. 81; Lim, C. Y./Tan, P. M. S. (2009), S. 296/300; Tirole, J. (2006), S. 362. 
ist es nicht grundsätzlich gesichert, dass Großaktionäre Vorstände oder Aufsichtsräte angemessen überwachen wollen und können. ${ }^{916}$

\subsubsection{Vorteilsnahmen durch Großaktionäre}

Die Treuepflicht zwischen den Aktionären einer Gesellschaft verpflichtet Großaktionäre nicht, Vorstände überhaupt oder gar besonders genau zu überwachen. ${ }^{917}$ Diese fehlende Pflicht von Großaktionären ist ein wesentlicher Unterschied zu Aufsichtsräten, die zur Vorstandsüberwachung gesetzlich verpflichtet $\operatorname{sind}^{918}$ und bei Pflichtverletzungen gegenüber den Aktiengesellschaften Schadensersatz leisten müssen. ${ }^{919}$ Folglich üben Großaktionäre ihren Einfluss auf Vorstände nur dann im Sinne aller Aktionäre aus, falls ihre Interessen mit den Interessen der restlichen Aktionäre übereinstimmen. ${ }^{920}$ Ohne diese Interessenharmonie haben opportunistische Großaktionäre Anreize, Kleinaktionäre zu übervorteilen, ${ }^{921}$ indem sie bspw. für Unternehmensstrategien werben, die ihren Risikopräferenzen entsprechen, oder mit Vorständen implizite Nebenabreden treffen. ${ }^{922}$ Erfahren Großaktionäre in vertraulichen Vorstandsgesprächen von abschlusspolitischen Ergebnisspaltungen, können sie ihre Aktien verkaufen, bevor deren Kurse aufgrund des allgemeinen Bekanntwerdens von abschlusspolitischen Ergebnisspaltungen sinken. ${ }^{923}$ Derartige gesetzlich verbotene Insidergeschäfte ${ }^{924}$ sind mit der Annahme von opportunistischen Agenten durchaus vereinbar, da die Annahme auch Regel- und Gesetzesbrüche wie „Lügen, Stehlen und Betrügen““925 abdeckt. ${ }^{926}$ Zudem ist es ungewiss, ob derartige Insidergeschäfte jemals aufgedeckt und sanktioniert werden.

Obwohl opportunistische Großaktionäre Anreize haben, Kleinaktionäre zu übervorteilen, kann aus zwei Gründen bezweifelt werden, dass Großaktionäre hierzu abschlusspolitische Ergebnisspaltung verwenden werden. Erstens lohnen sich riskante Insidergeschäfte für

916 Vgl. Engelen, C. (2015), S. 934 f. Ungefähr Denis, D. K. (2001), S. 204.

917 Die Treuepflicht befriedigt gemäß Cahn, A./Schild von Spannenberg, M. A. (2019), 53a AktG, Tz. 36 das „Bedürfnis nach einer inhaltlichen Kontrolle gesellschaftsbezogenen Verhaltens der Gesellschaftsorgane oder einzelner Aktionäre noch unterhalb der Schwelle des Rechtsmissbrauchs.“

918 Siehe $\S 111$ Abs. 1 AktG. Dazu Bress, S. (2008), S. 52.

919 Siehe den Unterabschnitt 6.1.3.

920 Ähnlich Engelen, C. (2015), S. 934 f.; Köke, J. (2004), S. 56.

921 Vgl. Ampenberger, M. (2010), S. 73; La Porta, R. et al. (2000), S. 4; Lim, C. Y./Tan, P. M. S. (2009), S. 296; Shleifer, A./Vishny, R. W. (1997), S. 758.

922 Vgl. Köke, J. (2004), S. 56; Shleifer, A./Vishny, R. W. (1997), S. 760. Weitere Beispiele bei La Porta, R. et al. (2000), S. 4; Lim, C. Y./Tan, P. M. S. (2009), S. 301; Shleifer, A./Vishny, R. W. (1997), S. 758-760; Tirole, J. (2006), S. 42. Jenkinson, T./Ljungqvist, A. (2001), S. 429 konstatieren: „If the US/UK corporate governance problem is one of ,strong managers, weak owners ' [...], in continental Europe, and many other countries, the corporate governance problem is rather one of ,strong block owners, weak minorities'."

923 Vgl. Almaleeh, N. M. S. (2019), S. 33. Allgemeiner Kleinmanns, H. (2016), S. 343 f.; Tirole, J. (2006), S. 362 .

924 So $\S \S 12$ Satz $1 \mathrm{Nr}$. 1; 13 Abs. 1 Satz 1; 14 Nr. 1 WpHG (i.d.F. vom 16. Juli 2007).

925 Williamson, O. E. (1990), S. 54.

926 Hierzu Wentges, P. (2002), S. 38. 
Großaktionäre nur dann, wenn diese relativ zu deren Aktienbesitz signifikante Volumina erreichen. Mit den Volumina von Aktienverkäufen oder -käufen steigt für Großaktionäre aber das Risiko, dass etwaige Insidergeschäfte bspw. durch die BaFin aufgedeckt werden. Zweitens haben Großaktionäre, abgesehen von riskanten Insidergeschäften, keinen Nutzen aus abschlusspolitischen Ergebnisspaltungen. Selbst wenn Großaktionäre die tatsächliche Ertragslage in Erfahrung bringen können, entstehen ihnen aus abschlusspolitische Ergebnisspaltungen, wenn sie aufgrund von diesen bspw. bereits ausgearbeitete Analysen anpassen müssen, Monitoring Expenditures. Insbesondere Großaktionäre wie Investmentoder Pensionsfonds, die zumeist Aktien von vielen verschiedenen Unternehmen besitzen, dürften diese teilweise unnötigen Monitoring Expenditures vermeiden wollen und von Vorständen eine unverzerrte Rechnungslegung verlangen.

\subsubsection{Eindämmung der Free-Rider-Problems}

Befinden sich Aktiengesellschaften im ausschließlichen Besitz von Kleinaktionären, können hinsichtlich der unmittelbaren Überwachung der Vorstände durch Kleinaktionäre ${ }^{927}$ Free-Rider-Problems auftreten. ${ }^{928}$ Bei Free Riders oder Trittbrettfahrern handelt es sich allgemein formuliert um Personen, welche die Nutzen von Gütern erlangen, ohne für diese zu bezahlen. ${ }^{929}$ Bei Aktiengesellschaften, die sich im ausschließlichen Eigentum von Kleinaktionären befinden, bestehen die Free-Rider-Problems darin, dass die Monitoring Expenditures der einzelnen Kleinaktionäre für die unmittelbare Vorstandsüberwachung höher sind als deren individuelle Nutzen, weshalb alle Kleinaktionäre als Free Rider von der Vorstandsüberwachung durch andere Kleinaktionäre profitieren wollen. ${ }^{930}$ Verfolgen jedoch alle Kleinaktionäre diese Strategie, werden Vorstände von keinem Aktionär unmittelbar überwacht. ${ }^{931}$ Diese Überwachungsdefizite können Vorstände unter anderen für abschlusspolitische Ergebnisspaltungen nutzen. ${ }^{932}$

Großaktionäre können Free-Rider-Problems eindämmen, da deren Nutzen aus einer genaueren Überwachung der Vorstände in einem günstigeren Verhältnis zu den hierfür

927 Siehe zur mittelbaren Überwachung der Vorstände durch Aufsichtsräte den Abschnitt 6.1.

928 Vgl. Hermalin, B. E./Weisbach, M. S. (2003), S. 10; Shleifer, A./Vishny, R. W. (1997), S. 741/765. Ebenso Bress, S. (2008), S. 47.

929 So Mankiw, N. G./Taylor, M. P. (2018), S. 306. Sinngemäß Barnea, A./Haugen, R. A./Senbet, L. W. (1981), S. 12.

930 Siehe Feser, N. (2009), S. 15; Hart, O. (1995a), S. 127; Hart, O. (1995b), S. 681; Shleifer, A./Vishny, R. W. (1986), S. 461 f.; Shleifer, A./Vishny, R. W. (1997), S. 764; Stratmann, J. (2005), S. 160; Walz, W. R. (1993), S. 99. Grundlegend Grossman, S. J./Hart, O. D. (1980), S. 42/59. Gemäß Bebchuk, L. A. (1989), S. 1839; Easterbrook, F. H./Fischel, D. R. (2001), S. 66; Ruhnke, K. (2000), S. 25; Ruhnke, K./Simons, D. (2018), S. 76; Walz, W. R. (1993), S. 98 f. erklären ungünstige Kosten-Nutzen-Verhältnisse bei der Vorstandsüberwachung die sog. rationale Apathie von Kleinaktionären.

931 Vgl. Feser, N. (2009), S. 15; Hart, O. (1995a), S. 127; Hart, O. (1995b), S. 681; Shleifer, A./Vishny, R. W. (1997), S. 741; Wentges, P. (2002), S. 81. Berens, E. (1988), S. 339 notiert: „Je mehr Aktionäre, um so größer die Macht des Vorstandes."

932 Allgemeiner Shleifer, A./Vishny, R. W. (1997), S. 741 f. 
erforderlichen Monitoring Expenditures steht. ${ }^{933}$ Überwachen Großaktionäre Vorstände aufgrund dieser günstigeren Kosten-Nutzen-Verhältnisse genauer, können hiervon auch Kleinaktionäre profitieren, da die Monitoring Expenditures für die Vorstandsüberwachung alleine von den Großaktionären getragen werden. ${ }^{934}$ Folglich können Kleinaktionäre entweder ihre Überwachungsaktivitäten und somit ihre Monitoring Expenditures senken oder erhalten, falls sie Vorstände bislang nicht überwacht haben, ${ }^{935}$ die Überwachungsaktivitäten der Großaktionäre kostenlos.

\subsubsection{Zwischenfazit}

In den vorherigen Unterabschnitten wurde untersucht, ob Großaktionäre Vorstände von abschlusspolitischen Ergebnisspaltungen abhalten wollen. Als Zwischenfazit ist festzuhalten, dass Insidergeschäfte der Großaktionäre diesem Willen entgegenstehen können. Abgesehen von illegalen Insidergeschäften, deren Entdeckungsrisiken mit den Beteiligungsquoten von Großaktionären tendenziell steigen, entstehen Großaktionären aus abschlusspolitischen Ergebnisspaltungen jedoch Monitoring Expenditures, die teilweise vermeidbar sind, falls Großaktionäre Vorstände aufgrund ihres Einflusses zu einer unverzerrten Rechnungslegung bewegen können. In diesem Fall lindern Großaktionäre, deren KostenNutzen-Verhältnisse aus der Vorstandsüberwachung mit steigenden Beteiligungsquoten immer günstiger werden, Free-Rider-Problems der Kleinaktionäre. In den folgenden Unterabschnitten wird untersucht, ob Großaktionäre Vorstände von abschlusspolitischen Ergebnisspaltungen abhalten können.

\subsubsection{Formaler und realer Einfluss}

Um abschlusspolitische Ergebnisspaltungen zu verhindern, reicht der diesbezügliche Wille der Großaktionäre nicht aus, sondern sie müssen zusätzlich über genug Einfluss auf Vorstände oder Aufsichtsräte verfügen. ${ }^{936}$ Der Einfluss der Großaktionäre auf Vorstände oder Aufsichtsräte aufgrund von Stimmrechten kann zwei Ursachen haben. Formaler Einfluss resultiert aus Stimmrechten, mit denen Großaktionäre ohne fremde Unterstützung bestimmte Entscheidungen, wie etwa die Bestellungen von Aufsichtsräten, ${ }^{937}$ herbeiführen oder bestimmte Entscheidungen, wie etwa Entlastungen der Vorstände, ${ }^{938}$ zumindest verhindern können. ${ }^{939}$ Dagegen resultiert realer Einfluss aus der Möglichkeit, andere stimm-

933 Hierzu Ampenberger, M. (2010), S. 73; Shleifer, A./Vishny, R. W. (1986), S. 462 f.; Shleifer, A./Vishny, R. W. (1997), S. 754; Stratmann, J. (2005), S. 160. Allgemeiner Berens, E. (1988), S. 334. Auch Köke, J. (2004), S. 55 f.; La Porta, R. et al. (1998), S. 1116.

934 Vgl. Grossman, S. J./Hart, O. D. (1980), S. 42/59; Shleifer, A./Vishny, R. W. (1986), S. 463. Ähnlich Ampenberger, M. (2010), S. 73; Engelen, C. (2015), S. 934 f.; Kleinmanns, H. (2016), S. 343-345.

935 Hierzu Fama, E. F. (1980), S. 291-293.

936 Im Allgemeinen optimistisch Engelen, C. (2015), S. 935.

937 Siehe $\$ 101$ Abs. 1 Satz 1 AktG.

938 Siehe $\$ 120$ Abs. 1 Satz 1 AktG.

939 Siehe Tirole, J. (2006), S. 36 f./334. 
berechtigte Aktionäre von einer Ausübung ihrer Stimmrechte im Interesse der Großaktionäre zu überzeugen, sodass die Großaktionäre mit dieser fremden Unterstützung bestimmte Beschlüsse herbeiführen oder zumindest verhindern können. ${ }^{940}$ Während der formale Einfluss von Großaktionären unmittelbar mit deren Beteiligungsquoten steigt, ist dieser Zusammenhang mittelbar auch beim realen Einfluss zu vermuten, da die Überzeugungskraft von Großaktionären tendenziell mit deren Beteiligungsquoten steigt. ${ }^{941}$

\subsubsection{Exit And Voice}

Neben dem formalen und realen Einfluss aus eigenen oder fremden Stimmrechten können Großaktionäre Vorstände oder Aufsichtsräte mit dem Konzept des ,,[E]xit [A]nd [V]oice“ ${ }^{\text {‘42 }}$ beeinflussen. ${ }^{943}$ Das Konzept des Exit And Voice besagt allgemein, dass unzufriedene Kunden die leistenden Unternehmen entweder wirtschaftlich sanktionieren, indem sie die Geschäftsbeziehungen mit den Unternehmen beenden (Exit), oder diese politisch sanktionieren, indem sie die Unternehmen kritisieren (Voice). ${ }^{944}$ Bezogen auf Aktionäre, die aufgrund von abschlusspolitische Ergebnisspaltungen unzufrieden sind, bedeutet das Konzept des Exit And Voice, dass Aktionäre ihre Aktien entweder verkaufen oder Vorstände bzw. Aufsichtsräte kritisieren können. ${ }^{945}$ Grundsätzlich können unzufriedene Aktionäre zwischen dem Verkauf ihrer Aktien oder der Kritik an den Vorständen bzw. Aufsichtsräten frei wählen. ${ }^{946}$ Die Nutzen aus den beiden Rechten hängen jedoch vom Umfang des Aktienbesitzes ab. Für unzufriedene Kleinaktionäre ist der Exit regelmäßig attraktiver als die Voice-Option, da Vorstände bzw. Aufsichtsräte die Kritik von Kleinaktionären zumeist kaum berücksichtigen und Kleinaktionäre ihre wenigen Aktien typischerweise zeitnah ohne wesentliche Preisabschläge verkaufen können. ${ }^{947}$ Dagegen ist für Großaktionäre die Voice- regelmäßig attraktiver als die Exit-Option, da Vorstände bzw. Aufsichtsräte die Kritik von Großaktionären zumeist berücksichtigen und Großaktionäre ihre Aktienpakete typischerweise zeitnah lediglich mit Preisabschlägen verkaufen können. Die eingeschränkten Exit-Optionen von Großaktionären können im Übrigen ein

940 Vgl. Kleinmanns, H. (2016), S. 342; Tirole, J. (2006), S. 36 f./334. Weiterführend analysieren Köke, J. (2004), S. 57 f./60-62; Lim, C. Y./Tan, P. M. S. (2009), S. 296/300 die Prinzipal-Agenten-Probleme zwischen Klein- und Großaktionären, wenn Großaktionäre aufgrund von pyramidenförmigen Konzernstrukturen über mehr Kontroll- als Eigentumsrechte verfügen.

941 Vgl. Campbell, T. S./Kracaw, W. A. (1980), S. 864/874; Leland, H. E./Pyle, D. H. (1977), S. 383 f.

942 Hirschman, A. O. (1970), S. 15.

943 Vgl. Kleinmanns, H. (2016), S. 342.

944 Grundlegend Hirschman, A. O. (1970), S. $4 / 15$ f.

945 Hierzu Kleinmanns, H. (2016), S. 342; Tirole, J. (2006), S. 334.

946 Gemäß Bayer, W. (2019), § 68 AktG, Tz. 34; Koch, J. (2020a), § 68 AktG, Tz. 10; Lange, K. W. (2019), $\S 68 \mathrm{AktG}, \mathrm{Tz} .6$ sind Aktien grundsätzlich frei übertragbar. Allerdings kann die Übertragbarkeit durch Vinkulierungen gemäß $\S 68$ Abs. 2 Satz 1 AktG sowie die Verkehrsfähigkeit durch Nebenverpflichtungen der Aktionäre gemäß $\S 55 \mathrm{Abs}$. 1 Satz $1 \mathrm{Akt} G$ eingeschränkt sein. Die Teilnahme- und Rederechte der Aktionäre auf Hauptversammlungen sind im Aktiengesetz nicht explizit normiert, gelten gemäß Hoffmann, J. (2019), § 118 AktG, Tz. 8/11 f.; Kubis, D. (2018), § 118 AktG, Tz. 38/53/74 f.; Liebscher, T. (2019a), § 118 AktG, Tz. 9 f. aber als selbstverständlich.

947 Sinngemäß Stratmann, J. (2005), S. 155. 
weiterer Grund dafür sein, dass Großaktionäre Vorstände bzw. Aufsichtsräte genauer als die Kleinaktionäre überwachen wollen. ${ }^{948}$ Aber selbst wenn Großaktionäre ihre Aktien zeitnah mit Preisabschlägen verkaufen, können diese Verkäufe für Vorstände nachteilig sein, sofern deren Vergütungen und Weiterbeschäftigungen von den Aktienkursen abhängen. Zudem können Kleinaktionäre Aktienverkäufe von Großaktionären als Signale für schlechte Ertragsaussichten interpretieren, sodass die Kleinaktionäre ebenfalls ihre Aktien verkaufen ${ }^{949}$ und der Aktienkurs weiter sinkt. Somit können Großaktionäre mit der Voice-Option sowie der angedrohten oder gar ausgeübten Exit-Option Vorstände bzw. Aufsichtsräte beeinflussen und diese u. a. von abschlusspolitischen Ergebnisspaltungen abhalten.

\subsubsection{Zwischenfazit}

In den vorherigen Unterabschnitten wurde untersucht, ob Großaktionäre Vorstände von abschlusspolitischen Ergebnisspaltungen abhalten können. Als Zwischenfazit ist festzuhalten, dass der reale oder formale Einfluss von Großaktionären auf Vorstände bzw. Aufsichtsräte hierzu grundsätzlich geeignet ist. Während für unzufriedene Kleinaktionäre der Verkauf ihrer Aktien häufig effizienter ist, kann es sich für Großaktionäre lohnen, Vorstände bzw. Aufsichtsräte zu kritisieren. Wird diese Kritik nicht berücksichtigt, können Großaktionäre mit Aktienverkäufen drohen, die insbesondere für Vorstände nachteilig sein können. Während der formale Einfluss von Großaktionären unmittelbar mit deren Beteiligungsquoten steigt, ist dieser Zusammenhang mittelbar auch beim realen Einfluss sowie beim Einfluss durch Kritik und Aktienverkäufe zu vermuten.

\subsubsection{Gesamtfazit und Formulierung der Hypothese}

Im vorliegenden Abschnitt wurde untersucht, ob Großaktionäre Vorstände von abschlusspolitischen Ergebnisspaltungen abhalten wollen und können. Als Gesamtfazit ist festzuhalten, dass deren Wille abseits von illegalen Insidergeschäften grundsätzlich vorhanden ist, da abschlusspolitische Ergebnisspaltungen den Großaktionären teilweise vermeidbare Monitoring Expenditures verursachen. Ebenso kann von der Fähigkeit der Großaktionäre, abschlusspolitische Ergebnisspaltungen zu verhindern, grundsätzlich ausgegangen werden, da sie Vorstände bzw. Aufsichtsräte mit eigenen oder fremden Stimmrechten sowie mittels Kritik oder Aktienverkäufen beeinflussen können. Basierend auf diesen Erkenntnissen wird die folgende Alternativhypothese formuliert:

948 Siehe hierzu den Unterabschnitt 6.3.3.

949 Vgl. Campbell, T. S./Kracaw, W. A. (1980), S. 864; Leland, H. E./Pyle, D. H. (1977), S. 371. 
$\mathrm{H}_{2.3}$ : [Eigentümerstrukturen] Wenn Aktionäre über wesentliche Anteile verfügen, verhindern sie abschlusspolitische Ergebnisspaltungen mit aufgegebenen Geschäftsbereichen.

Die zugehörige Nullhypothese, die abgelehnt werden muss, um die obige Alternativhypothese annehmen zu können, lautet:

$\mathrm{H}_{2.3}$ : Wenn Aktionäre über wesentliche Anteile verfügen, verhindern sie keine abschlusspolitischen Ergebnisspaltungen mit aufgegebenen Geschäftsbereichen.

\subsection{Kapitalstrukturen}

\subsubsection{Zielsetzung}

Kapitalstrukturen von Unternehmen wurden in der Tab. 5 als interne Corporate-Governance-Mechanismen klassifiziert, wobei geeignete Kapitalstrukturen typischerweise mit hohen Verschuldungsgraden gleichgesetzt werden. Dahinter steht das Kalkül, dass sich Vorstände bei hohen Verschuldungsgraden zum einen mehr anstrengen, um Insolvenzen zu vermeiden, und zum anderen durch Fremdkapitalgeber genauer überwacht werden. ${ }^{950}$ Bezogen auf die Problemstellung in der vorliegenden Arbeit muss untersucht werden, ob hohe Verschuldungsgrade verhindern, dass Vorstände abschlusspolitische Ergebnisspaltungen vornehmen wollen. Die ebenfalls relevante Frage nach der diesbezüglichen Fähigkeit ist wie im Unterabschnitt 6.2.1 zu bejahen, da Vorstände für die Erstellung der Jahresbzw. Konzernabschlüsse und somit für etwaige abschlusspolitische Ergebnisspaltungen verantwortlich sind. Daher wird im Folgenden nur die Frage untersucht, ob Vorstände auf abschlusspolitische Ergebnisspaltungen verzichten wollen, wenn die von ihnen geleiteten Unternehmen hohe Verschuldungsgrade aufweisen.

\subsubsection{Verschuldungsgrade und Vorstände}

Mit der Verschuldung von Unternehmen steigen deren Insolvenzrisiken. Einerseits arbeiten Vorstände bei hohen Verschuldungsgraden typischerweise mehr und senken Kosten, da Insolvenzen deren Weiterbeschäftigungen, Vergütungsansprüche und Reputationen gefährden. ${ }^{951}$ Andererseits zwingen hohe Verschuldungsgrade Vorstände zu kurzfristigen Maßnahmen. da die Vorstände nicht wissen, ob sie langfristige, vorteilhaftere Maßnahmen vor einer etwaigen Insolvenz umsetzen können. ${ }^{952}$ Möglicherweise verbergen opportunistische Vorstände bei hohen Verschuldungsgraden mit abschlusspolitischen Ergebnisspaltungen

950 Vgl. Tirole, J. (2006), S. 20. Allgemeiner Bress, S. (2008), S. 49; DeFond, M. L. (1992), S. 21.

951 Siehe Ampenberger, M. (2010), S. 69; Bress, S. (2008), S. 49; DeAngelo, H./DeAngelo, L./Skinner, D. J. (1994), S. 114; Easterbrook, F. H./Fischel, D. R. (2001), S. 282; Nobach, K. (2006), S. 187; Tirole, J. (2006), S. 20/29.

952 Vgl. Almaleeh, N. M. S. (2019), S. 36; DeAngelo, H./DeAngelo, L./Skinner, D. J. (1994), S. 124/137. 
etwaige Probleme mit der Unternehmensstrategie, Ertragslage oder Finanzierung, damit Eigen- und Fremdkapitalgeber die Vorstände nicht durch niedrigere Vergütungen oder Abberufungen sanktionieren respektive den Unternehmen aufgrund von verletzten Kreditklauseln ihr Kapital entziehen. ${ }^{953}$ Im Ergebnis ist unklar, ob hohe Verschuldungsgrade die Interessen der Aktionäre und Vorstände angleichen, und zu bezweifeln, dass Vorstände bei hohen Verschuldungsgraden auf abschlusspolitische Ergebnisspaltungen verzichten.

\subsubsection{Verschuldungsgrade und Fremdkapitalgeber}

Aus der Sicht von Fremdkapitalgebern haben steigende Verschuldungsgrade vor allem zwei Konsequenzen. Zum einen wird es wahrscheinlicher, dass Unternehmen ihre Verbindlichkeiten gegenüber den Fremdkapitalgebern nicht erfüllen können. Zum anderen wird es für Vorstände mit steigenden Verschuldungsgraden attraktiver, Unternehmenswerte überproportional von den Fremdkapitalgebern auf die Aktionäre zu übertragen, ${ }^{954}$ da Vorstände außerhalb von Insolvenzen durch Aktionäre bzw. Aufsichtsräte stärker belohnt oder sanktioniert werden können als durch Fremdkapitalgeber. Beispielsweise können Vorstände hochriskante Geschäfte abschließen, die im ungünstigen Fall zu einem vollständigen Verlust der Einlagen der Aktionäre und zu vollständig entwerteten Forderungen der Fremdkapitalgeber führen. Im günstigen Fall bringen diese Geschäfte den Aktionären jedoch hohe Renditen ein, während die Forderungen der Fremdkapitalgeber lediglich in fixer Höhe beglichen werden müssen. ${ }^{955}$ Folglich können hohe Verschuldungsgrade Fremdkapitalgeber als Prinzipale der Vorstände dazu veranlassen, ihre Informationsasymmetrien gegenüber Vorständen abzubauen und diese ohne unmittelbare Vergütungen zu überwachen. ${ }^{956} \mathrm{Zu}$ untersuchen bleibt, ob Fremdkapitalgeber, die wie Aktionäre als Prinzipale fungieren, bei hohen Verschuldungsgraden dafür sorgen können, dass Vorstände als deren Agenten auf abschlusspolitische Ergebnisspaltungen verzichten. Vor diesem Hintergrund kommen ins-

953 Siehe DeAngelo, H./DeAngelo, L./Skinner, D. J. (1994), S. 121/124; DeFond, M. L./Jiambalvo, J. (1993), S. 418/428; DeFond, M. L./Jiambalvo, J. (1994), S. 147; Nobach, K. (2006), S. 188 f.; Ronen, J./Yaari, V. (2008), S. 14/20; Watts, R. L./Zimmerman, J. L. (1986), S. 216. Analog Günzer, E. (2016), S. 232; Quick, R./Wiemann, D. (2012), S. 1116-1118. Ungefähr Baber, W. R./Kumar, K. R./Verghese, T. (1995), S. 388. Küting, K. (2008), Teil H, Tz. 2107 argumentiert normativ: ,[D]ie Unternehmensleitung [muss] bemüht sein [...], die Unternehmenskrise zu verschleiern oder zumindest zu verharmlosen, um nicht 'Kredit' in jeglichem Wortsinn zu verspielen “ [Hervorhebung im Original nicht übernommen].

954 Analog bereits Fama, E. F./Miller, M. H. (1972), S. 151 f./169. Ebenso Black, F. (1976), S. 218; Black, F./Scholes, M. (1973), S. 651; DeFond, M. L. (1992), S. 21; Myers, S. C. (2001), S. 96; Smith jr., C. W./Warner, J. B. (1979), S. 153; Watts, R. L./Zimmerman, J. L. (1986), S. 189-191.

955 Grundlegende zu dieser Form der Risikoabwälzung Jensen, M. C./Meckling, W. H. (1976), S. 353 f. beschrieben. Später auch Barnea, A./Haugen, R. A./Senbet, L. W. (1981), S. 9; Ewert, R. (1990), S. 24; Shleifer, A./Vishny, R. W. (1997), S. 760; Simunic, D. A./Stein, M. T. (1987), S. 9. Weitere Möglichkeiten, wie Vorstände Fremdkapitalgeber zugunsten von Eigenkapitalgebern benachteiligen können, zeigen Palmrose, Z.-V. (1984), S. 232 f.; Smith jr., C. W./Warner, J. B. (1979), S. 118 f.

956 Ungefähr DeAngelo, H./DeAngelo, L./Skinner, D. J. (1994), S. 133 f.; Easterbrook, F. H./Fischel, D. R. (2001), S. 282; Köke, J. (2004), S. 56. Auf die Problematik, wenn Aktionäre durch Aufsichtsräte vertreten werden, wird im Unterabschnitt 6.1.1 eingegangen. 
besondere zwei Szenarien, die sich nicht gegenseitig ausschließen, für das Verhalten der Fremdkapitalgeber in Betracht.

Im ersten Szenario antizipieren die Fremdkapitalgeber, dass Vorstände bei hohen Verschuldungsgraden die tatsächliche Ertragslage möglicherweise mit abschlusspolitischen Ergebnisspaltungen verschleiern. ${ }^{957}$ Folglich können sich die Fremdkapitalgeber ihre höheren Informations- und Kreditrisiken vergüten lassen, indem sie höhere Risikoprämien und somit höhere Zinssätze verlangen. ${ }^{958}$ Die höheren Fremdkapitalkosten können Vorstände aus zwei Gründen zu einem Verzicht auf abschlusspolitische Ergebnisspaltungen motivieren. ${ }^{959}$ Zum einen sinkt mit steigenden Fremdkapitalkosten c. p. der Unternehmenserfolg, wodurch variable Vorstandsvergütungen typischerweise sinken. Zum anderen können Aktionäre hohe Fremdkapitalkosten tendenziell leichter als abschlusspolitische Ergebnisspaltungen erkennen und Aufsichtsräte bzw. Vorstände entsprechend sanktionieren. Um niedrigere variable Vergütungen und Sanktionen zu vermeiden, haben Vorstände bei hohen Verschuldungsgraden somit Anreize, auf abschlusspolitische Ergebnisspaltungen zu verzichten.

Im zweiten Szenario erkennen die Fremdkapitalgeber erst nach der Kreditvergabe, dass Vorstände die tatsächliche Ertragslage mit abschlusspolitischen Ergebnisspaltungen verschleiern. Da Fremdkapitalgeber Kreditvergaben kurzfristig zumeist nicht rückabwickeln oder die Zinsen anheben können, bauen sie typischerweise ihre Informationsasymmetrien gegenüber den Vorständen ab, um zumindest ihre tatsächlichen Kreditrisiken einschätzen zu können. Von dieser intensiveren Vorstandsüberwachung durch die Fremdkapitalgeber können auch die Aktionäre profitieren. Allerdings können Vorstände trotz einer intensiveren Überwachung durch die Fremdkapitalgeber abschlusspolitische Ergebnisspaltungen vornehmen. ${ }^{960} \mathrm{Da}$ Unternehmen mit hohen Verschuldungsgraden aber Kredite häufig verlängern oder neue Kredite aufnehmen müssen, können Fremdkapitalgeber bei den diesbezüglichen Verhandlungen höhere Risikoprämien verlangen, welche die im ersten Szenario beschriebenen Konsequenzen haben können. ${ }^{961}$ Folglich haben Vorstände bei hohen Verschuldungsgraden auch nach den Kreditvergaben Anreize, um auf abschlusspolitische Ergebnisspaltungen zu verzichten. ${ }^{962}$

\subsubsection{Fazit und Formulierung der Hypothese}

Im vorliegenden Abschnitt wurde untersucht, ob Vorstände aufgrund von hohen Verschuldungsgraden auf abschlusspolitische Ergebnisspaltungen verzichten. Als Fazit ist festzu-

957 Ähnlich Barnea, A./Haugen, R. A./Senbet, L. W. (1981), S. 9 f.; Quick, R. (2012), S. 248.

958 Sinngemäß Watts, R. L./Zimmerman, J. L. (1986), S. 186.

959 Vgl. Quick, R. (2012), S. 248.

960 Allgemeiner Tirole, J. (2006), S. 36.

961 Vgl. Shleifer, A./Vishny, R. W. (1997), S. 757. Sehr differenziert Diamond, D. W. (1991), S. 730-732.

962 Deutlich allgemeiner DeAngelo, H./DeAngelo, L./Skinner, D. J. (1994), S. 134. 
halten, dass die Insolvenzrisiken aus hohen Verschuldungsgraden Vorstände zu kurzfristigen Maßnahmen zwingen, die auch abschlusspolitische Ergebnisspaltung umfassen können. Da Fremdkapitalgeber bei höheren Verschuldungsgraden abschlusspolitische Ergebnisspaltungen aber entweder antizipieren und mit höheren Zinsen sanktionieren oder die Vorstände zumindest intensiver überwachen, werden abschlusspolitische Ergebnisspaltungen mit steigenden Verschuldungsgraden unattraktiver. Basierend auf diesen Erkenntnissen wird die folgende Alternativhypothese formuliert:

$\mathrm{H}_{2.4}$ : [Kapitalstrukturen] Je höher die Verschuldungsgrade, desto schwächer sind abschlusspolitische Ergebnisspaltungen mit aufgegebenen Geschäftsbereichen.

Die zugehörige Nullhypothese, die abgelehnt werden muss, um die obige Alternativhypothese annehmen zu können, lautet:

$\mathrm{HO}_{2.4}$ : Je höher die Verschuldungsgrade, desto stärker sind abschlusspolitische Ergebnisspaltungen mit aufgegebenen Geschäftsbereichen bzw. es gibt keinen diesbezüglichen Zusammenhang.

Dieses Kapitel wird unter der Creative Commons Namensnennung 4.0 International Lizenz (http://creativecommons.org/licenses/by/4.0/deed.de) veröffentlicht.

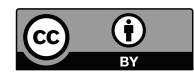

